\title{
Multiscale Modeling of Poly(lactic acid) Production: From Reaction Conditions to Rheology of Polymer Melt
}

\author{
Zubov, Alexandr; Sin, Gürkan
}

Published in:

Chemical Engineering Journal

Link to article, DOI:

10.1016/j.cej.2017.12.033

Publication date:

2018

Document Version

Peer reviewed version

Link back to DTU Orbit

Citation (APA):

Zubov, A., \& Sin, G. (2018). Multiscale Modeling of Poly(lactic acid) Production: From Reaction Conditions to Rheology of Polymer Melt. Chemical Engineering Journal, 336, 361-375.

https://doi.org/10.1016/j.cej.2017.12.033

\section{General rights}

Copyright and moral rights for the publications made accessible in the public portal are retained by the authors and/or other copyright owners and it is a condition of accessing publications that users recognise and abide by the legal requirements associated with these rights.

- Users may download and print one copy of any publication from the public portal for the purpose of private study or research.

- You may not further distribute the material or use it for any profit-making activity or commercial gain

- You may freely distribute the URL identifying the publication in the public portal

If you believe that this document breaches copyright please contact us providing details, and we will remove access to the work immediately and investigate your claim. 


\section{Accepted Manuscript}

Multiscale Modeling of Poly(lactic acid) Production: From Reaction Conditions to Rheology of Polymer Melt

Alexandr Zubov, Gürkan Sin

PII:

S1385-8947(17)32145-9

DOI: https://doi.org/10.1016/j.cej.2017.12.033

Reference: CEJ 18194

To appear in:

\section{Chemical Engineering Journal}

Received Date: $\quad 16$ October 2017

Revised Date: $\quad 4$ December 2017

Accepted Date: 6 December 2017

Please cite this article as: A. Zubov, G. Sin, Multiscale Modeling of Poly(lactic acid) Production: From Reaction Conditions to Rheology of Polymer Melt, Chemical Engineering Journal (2017), doi: https://doi.org/10.1016/j.cej. 2017.12.033

This is a PDF file of an unedited manuscript that has been accepted for publication. As a service to our customers we are providing this early version of the manuscript. The manuscript will undergo copyediting, typesetting, and review of the resulting proof before it is published in its final form. Please note that during the production process errors may be discovered which could affect the content, and all legal disclaimers that apply to the journal pertain. 


\title{
Multiscale Modeling of Poly(lactic acid) Production: From Reaction Conditions to Rheology of Polymer Melt
}

\author{
Alexandr Zubov*, Gürkan Sin
}

Process and Systems Engineering Center (PROSYS), Department of Chemical and Biochemical Engineering, Technical University of Denmark, Søltofts Plads 229, 2800 Kgs. Lyngby, Denmark

*Corresponding author's e-mail address: azub@kt.dtu.dk

\begin{abstract}
Poly(L-lactic acid) (PLLA) is a fully biodegradable bioplastic with promising market potential. The paper deals with systematic development and analysis of the modeling framework allowing direct mapping between PLLA production process conditions and rheological properties of the polymer melt. To achieve this, the framework builds upon three distinct elements that approach the production process from different scales: (i) macroscopic deterministic model of L,L-lactide ring opening polymerization taken from the literature, (ii) microscopic stochastic simulation of the polymerization process based on hybrid Monte Carlo approach, and (iii) mesoscopic public domain model of polymer chain reptation dynamics. Based on the input reaction conditions, the macro-scale model predicts L,L-lactide conversion and averaged molar mass of PLLA, while the micro-scale and meso-scale simulations allow prediction of full molar mass distribution and melt viscosity of the product. The developed predictive tool is validated by literature data, i.e. experimentally measured rheological characteristics of three commercial PLLA samples with different molecular architecture. Moreover, comprehensive global sensitivity analysis has been carried out to support exploration of the process conditions space in relation to target polymer melt properties. Computational efficiency of the developed model achieved so far foreshadows its potential use as soft sensor for molar mass distribution and melt viscosity in the optimization and control of PLLA production.
\end{abstract}

Keywords: Poly(lactic) acid; Mathematical modeling; Monte Carlo simulation; Polymer melt rheology; Global sensitivity analysis; Identifiability analysis. 


\section{Introduction}

Poly(lactic acid) (PLA), or poly(lactide) is a fully biodegradable thermoplastic polyester that is produced from renewable resources and offers significantly reduced carbon footprint when compared to its petroleumbased market competitors [1]. Although PLA was first synthesized more than 80 years ago, its use was limited only to biomedical applications due to its high cost. The breakthrough came in 1990s with the discovery of commercially feasible way of high molecular weight PLA production [2], enabling better control over the production process and extending the applications of PLA to packaging, textile industry, automotive parts, insulation foams and many others $[3,4,5,6]$. Nowadays, PLA is the most widely produced renewable and biodegradable polymer, having the third biggest market share among bioplastics (after the groups of cellulose-based and starch-based biopolymers) [7], and steep increase in worldwide production capacity predicted for near future [8].

Monomer for PLA - optically active lactic acid - is obtained by fermentation of simple carbohydrates [9]. The industrial production of high molecular weight PLA is usually carried out by the ring opening polymerization (ROP) of lactide, a cyclic diester of latic acid using a tin(II)octoate catalyst, $\mathrm{Sn}(\mathrm{Oct})_{2}$ [10]. During the ROP process, molecular architecture of the polymer can be controlled via proper selection of the co-catalyst, addition of comonomers and/or grafting/cross-linking agents [11,12], and by modifying the reaction conditions, resulting in a product with improved technical properties [13]. Moreover, purity and chirality of the lactide monomer have direct impact on the polymerization kinetics and product chirality, respectively, affecting the manufacturing process and product properties: for instance, carboxylic acids present in lactide contribute to the catalyst deactivation and therefore decrease the initial rate of polymerization [14]; chirality of PLA determines its crystallization kinetics and thus thermal properties such as glass transition temperature $T_{\mathrm{g}}[15]$.

Main objective of this paper is the development of a multi-scale integrated modeling framework and its computational implementation as in silico design tool for new PLA-based materials and tailoring of their rheological properties. Such computational tool is based on the following elements: (i) 
mathematical model of poly-L-lactide (PLLA) production enabling mapping between polymerization conditions and product application (rheological) properties, (ii) set of reliable rheological experimental data for validation of the developed model, and (iii) model sensitivity analysis enabling thorough understanding of the model behavior and contribution of individual process/model parameters to the predicted polymer quality. Let us review information on these three subjects published in the literature.

Mechanism of L,L-lactide ROP has been extensively discussed $[14,16,17]$ and it is now widely accepted that the initiation of the reaction is carried out via the "alkoxide initiation mechanism". According to this theory, stannous octoate $\mathrm{Sn}(\mathrm{Oct})_{2}$ reacts with protic (i.e., OH-bearing) species to form an alkoxide, which is the true species initiating the polymerization. $\mathrm{Sn}(\mathrm{Oct})_{2}$ and $\mathrm{OH}$-bearing species (e.g., water or alcohol) are usually termed initiator and co-initator, or catalyst and co-catalyst, respectively [18]. The system exhibits the so-called living behavior, meaning that the equilibrium between dormant and active (growing) chains is established rapidly and the reaction proceeds at a constant number of growing chains, while their chain length (and molar mass) distribution is evolving during the process due to various elementary reactions (transesterification, chain scission etc.). The general scheme of elementary reactions involved in L,L-lactide ROP in the presence of $\mathrm{Sn}(\mathrm{Oct})_{2}$ and arbitrary alcohol (ROH) as presented by Kowalski et al. [17] is displayed in Figure 1. 


$$
\begin{aligned}
& \mathrm{Sn}(\mathrm{Oct})_{2}+\mathrm{ROH} \leftrightarrow \mathrm{OctSnOR}+\mathrm{OctH} \\
& \mathrm{OctSnOR}+\mathrm{ROH} \leftrightarrow \mathrm{Sn}(\mathrm{OR})_{2}+\mathrm{OctH} \\
& \ldots-\mathrm{SnOR}+\mathrm{nLA} \rightleftarrows \ldots-\mathrm{SnO}-(\mathrm{la})_{n}-\mathrm{R} \\
& \ldots-\mathrm{SnO}-(\mathrm{la})_{n}-\mathrm{R}+\mathrm{OctH} \rightleftarrows \\
& \ldots-\text { SnOct }+\mathrm{HO}-(\mathrm{la})_{n}-\mathrm{R} \\
& \ldots-\mathrm{SnO}-(\mathrm{la})_{n}-\mathrm{R}+\mathrm{ROH} \rightleftharpoons \\
& \ldots-\mathrm{SnOR}+\mathrm{HO}-(\mathrm{la})_{n}-\mathrm{R} \\
& \ldots-\mathrm{Sn}-(\mathrm{la})_{n}-\mathrm{R}+\mathrm{OctH} \rightarrow \ldots-\mathrm{SnOH}+\mathrm{Oct}-(\mathrm{la})_{n}-\mathrm{R} \\
& \ldots-\mathrm{SnOH}+\mathrm{nLA} \rightleftarrows \ldots-\mathrm{SnO}-(\mathrm{la})_{n}-\mathrm{H} \\
& \ldots-\mathrm{SnO}-(\mathrm{la})_{n}-\mathrm{H}+\mathrm{OctH} \leftrightarrow \\
& \ldots-\text { SnOct }+\mathrm{HO}-(\mathrm{la})_{n}-\mathrm{H} \\
& \ldots-\mathrm{SnO}-(\mathrm{la})_{n}-\mathrm{H}+\mathrm{ROH} \rightleftharpoons \\
& \ldots-\mathrm{SnOR}+\mathrm{HO}-(\mathrm{la})_{n}-\mathrm{H} \\
& \ldots-\mathrm{SnO}-(\mathrm{la})_{n}-\mathrm{H}+\mathrm{OctH} \rightarrow \\
& \ldots-\mathrm{SnOH}+\text { Oct }-(\mathrm{la})_{n}-\mathrm{H}
\end{aligned}
$$

Figure 1. Typical reactions involved in ring opening polymerization of L,L-lactide. Symbol "Oct" represents the octoate (2-ethylhexanoate) group, "LA" denotes molecule of monomer (L,L-lactide) while "la" represents the repeating (lactoyl) unit. Taken from [17].

Mathematical models of PLLA production using $\mathrm{Sn}(\mathrm{Oct})_{2}$ as a catalyst published in literature vary greatly in their complexity. Puaux et al. [19] presented relatively simple kinetic scheme (omitting chain transfer, transesterification and chain scission reactions) while receiving only poor agreement with experimental data for average polymer molar masses. Mehta et al. [20] developed model of similar complexity to Puaux, but compared two different models of termination: (i) chain transfer to monomer, and (ii) cationic ROP (interand intramolecular transfer). In a series of papers, Yu et al. $[18,21]$ presented an experimentally validated model for L,L-lactide ROP using $\mathrm{Sn}(\mathrm{Oct})_{2}$ and dodecan-1-ol as a catalyst and co-catalyst, resp., providing predictions in a wide range of operating conditions. Notably, the model included reversible chain transfer, intermolecular transesterification and chain scission reactions. In the last paper of this series [22], the method of solving the population balances was extended from polymer moments to the computationally efficient method of "fractionated moments", enabling approximate reconstruction of full molar mass distribution of 
the polymer from moments calculated for two different categories of chains, based on the reaction steps they experienced. The kinetic scheme [21] was also used by other authors [23] to model their own batch ROP of L,L-lactide, obtaining agreement with experiments and similar estimated values of model parameters. Sosnowski et al. [24] studied an influence of the intermolecular transesterification ("redistribution") reactions on PLA molecular weight using a simple Monte Carlo simulation and compared the predictions with measured MALDI-TOF spectra. Weng et al. [25] developed and experimentally validated model of L,Llactide and $\varepsilon$-caprolactam copolymerization at $140^{\circ} \mathrm{C}$, while introducing parallel encounter pair model of diffusion limitation [26] for propagation and transesterification reaction rates at higher conversions, when the reaction mixture becomes more viscous due to high polymer chain length. Last but not least, group of Kiparissides developed probably kinetically most complex model for L,L-lactide ROP in the presence of water traces, which included several reaction steps neglected in other works (intramolecular transesterifications, formation of cyclic chains, ester end-group formation and others). The model was successfully applied to predict conversion and average molar masses in the case of polymerizations initiated by water [27] and various polyalcohols [28]. Recently, the model was extended by Monte Carlo simulation in order to predict full molar mass distribution (MMD) of PLA [29].

Rheology of PLA is important not only during the polymerization and for the product downstream processing (e.g., by extrusion, injection molding or fiber spinning), but also due to the fact that measurements of PLA melt viscosity can provide direct evidence for changes in the product quality (loss of molecular weight) due to improper stabilization against residual moisture present in polymer [30]. Therefore rheology of optically pure or mixed PLA polymers as well as their copolymers and blends has been studied extensively. Pioneering study of effect of molar mass and temperature on melt viscosity of PLLA was conducted by Cooper-White and Mackay [31], who concluded that - at a given temperature - PLLA requires significantly higher molecular weight to exhibit similar melt viscoelastic behavior as conventional polymers such as polystyrene. Concerning the rheological behavior of PLA, probably the most active research has been carried out by the group of Dorgan who investigated entanglement and chain architecture effects in PLA [32], rheology of commercial-grade samples [33], blends of PLA with different molecular architecture 
[34] and solution and single-chain properties of homopolymers and copolymers spanning wide ranges of molecular weight and stereoisomer ratios [35].

With the increasing computational power, the uncertainty and sensitivity analyses of mathematical models are receiving increasing attention in science and engineering, since they can bring insight to the behavior of complex models, facilitate parameter estimation, and provide valuable (in some cases necessary) statistical information about the uncertainty in model predictions [36]. Unfortunately, in the field of polymer reaction engineering, the use of such methods is limited mainly to the "manual investigation", i.e., observing effect of manual step change in the input parameter values on the simulation output. Among rigorous methods, only local sensitivity (also termed "one factor at a time", OAT) analysis has been reported in the literature on polymerization modeling. Such works include for example batch polymerization of methyl methacrylate (MMA) [37], styrene/acrylonitrile copolymerization in a tubular reactor [38], reversible-deactivation polymerization [39], thermopolymerization of furfuryl methacrylate [40] or semi-batch emulsion polymerization of styrene [41]. In several works, OAT analysis has been also employed in model parameter estimation [42,43,44]. Apart from OAT approach, Bojarski et al. [45] carried out global sensitivity analysis of batch styrene emulsion polymerization model using Monte Carlo sampling. Surprisingly, as a measure of sensitivity they used standardized regression coefficients (SRCs), which are applicable only for linear models, although their results indicate that the model is far from being linear, especially in prediction of average polymer molar mass and polydispersity index. No other attempt to use rigorous global sensitivity analysis (GSA) methods in polymerization reaction modeling has been found in the literature so far. Note that here we strictly distinguish between the use of GSA as part of model development and model-based process optimization/control studies that can also bring insight into the influence of model inputs on its outputs, however at the expense of formulating and solving an optimization problem $[46,47]$.

Motivated by recent advances in the online monitoring and model-based control of industrial polymerization processes $[48,49,50]$, we aim at the development of robust and computationally efficient model of L,L-lactide ring opening polymerization for a potential use as a soft sensor for product MMD and melt viscosity in off-line and on-line optimization of the industrial production of PLA. For this purpose we 
define a hybrid modular framework which is based on data flow between (i) macro-scale batch reactor model [21], micro-scale hybrid Monte Carlo (MC) simulation of the process, and (iii) a tool for estimation of rheological properties of polymer melt with general branch-on-branch molecular architecture [51]. Results of the simulations are validated by the experimental data on PLLA melt rheology taken from the literature. The developed modeling framework is accompanied by comprehensive global sensitivity analysis, and the speed and accuracy of the predictions is critically assessed.

\section{Framework for Modeling of PLLA Production}

Multi-scale modeling has become an important part of polymer science and polymer reaction engineering, helping to understand the existing polymerization processes, design new materials and clarify structureproperty relationships in polymeric materials $[52,53,54]$. The multi-scale computational framework for mapping between reaction conditions in batch ROP reactor and PLLA application properties presented here is based on three fundamental parts illustrated in Fig. 2:

1. Macro-scale process model of ROP reactor is based on dynamic material and population balances represented by set of ordinary differential equations (ODEs). As the model inputs, initial and process conditions are provided (initial composition of the batch, reaction temperature). The model enables prediction of monomer conversion and average molar masses of polymer $\left(M_{n}, M_{w}\right)$ in time. Moreover, it provides profiles dynamic profiles of (i) concentrations of both non-polymeric and polymeric species, and (ii) reaction rates of elementary reaction steps in polymerization kinetic scheme, which are then used by the subsequent Monte Carlo simulation.

2. Micro-scale model of polymerization kinetics is following the well-known kinetic Monte Carlo (MC) simulation algorithm proposed by Gillespie [55], but in order to speed up the calculations, it utilizes profiles of some variables pre-calculated by macro-scale model (see previous paragraph). Therefore it can be called "Hybrid MC" simulation. Utilization of the pre-calculated profiles allows 
MC model to focus only on dynamics of polymeric species (non-polymeric being already covered by macro-scale model) which leads to significant reduction in a number of simulated molecules and thereby shorter simulation time. Its output is provided in the form of a table representing molecular architecture (i.e., chain length in case of linear polymerization) of statistical number of polymer chains, which can be translated into full molar mass distribution or distribution averages $\left(M_{n}, M_{w}\right)$.

3. Model of polymer chain arm reptation and retraction is based on tube theory of macromolecular chain dynamics [51] and is freely available under the name "BoB". Utilizing the information about structure of polymer chains predicted by $\mathrm{MC}$ model, $\mathrm{BoB}$ estimates typical rheological characteristics of viscoelastic polymer melts, such as shear rate dependency of loss and storage moduli.

More detailed information about the individual parts of the modeling framework and data flow between them is provided in the following subsections.
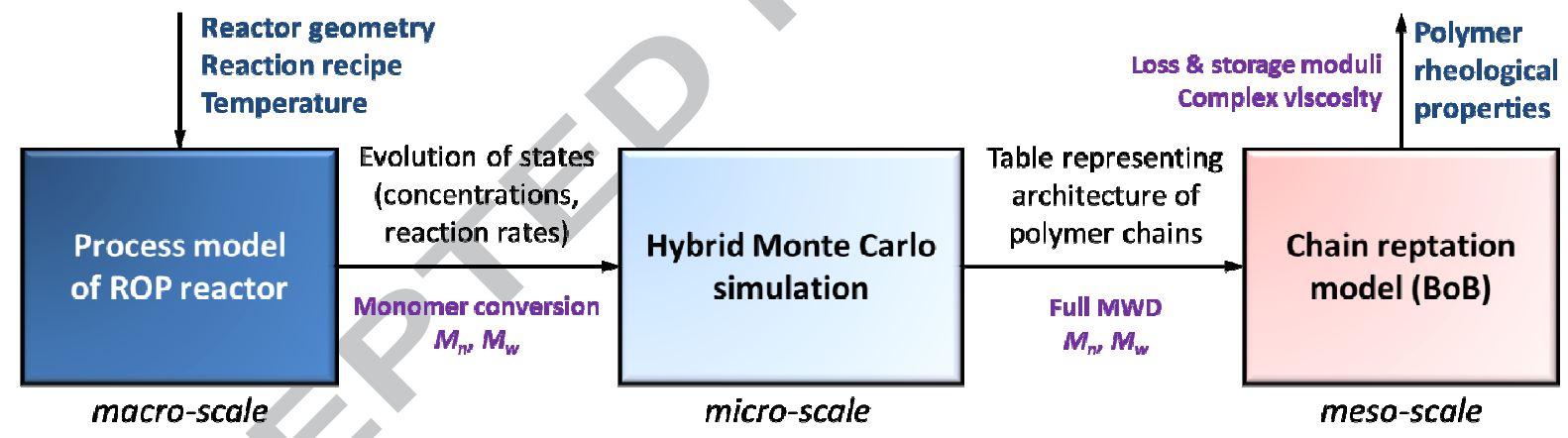

Figure 2. Illustration of three main pillars of the computational framework for modeling of PLLA production. The process/product characteristics that can be measured experimentally are marked by purple font.

\subsection{Deterministic Model}

For a deterministic simulation of L,L-lactide ring opening polymerization in a batch reactor we implemented the model of $\mathrm{Yu}$ et al. [21] due to reasonable degree of its complexity and versatility - it has been 
experimentally validated over a wide range of operating conditions (temperatures from 130 to $180^{\circ} \mathrm{C}$, monomer to catalyst ratio range of $1 \times 10^{4}-2 \times 10^{4}$, co-catalyst to catalyst ratio between 0.5 and 100). In this work, $\mathrm{Sn}(\mathrm{Oct})_{2}$ and dodecan-1-ol are used as catalyst and co-catalyst, respectively. Let us briefly summarize the model in the following paragraphs.

The assumed polymerization kinetic scheme is presented in Fig. 3 using the following symbols: $M$ for monomer (L,L-lactide) molecule, $C$ for catalyst (tin(II)octoate) molecule, $A$ for octanoic acid, and $R_{n}, D_{n}, G_{n}$ represent the active (growing), dormant (OH-bearing) and terminated (dead) chains composed of $n$ monomeric units, resp. Co-catalyst molecule (dodecan-1-ol) is formally denoted as $D_{0}$. Note that the propagation step means a polymer chain length increase by 2 units, since opening of lactide ring results in addition of 2 lactoyl units in a chain. It is evident from Fig. 3 that chain transfer, transesterification and chain scission reactions are assumed to have the same rate coefficient $\left(k_{s}, k_{t e}, k_{d e}\right.$, resp.) regardless of the reaction direction or type of the chain involved.

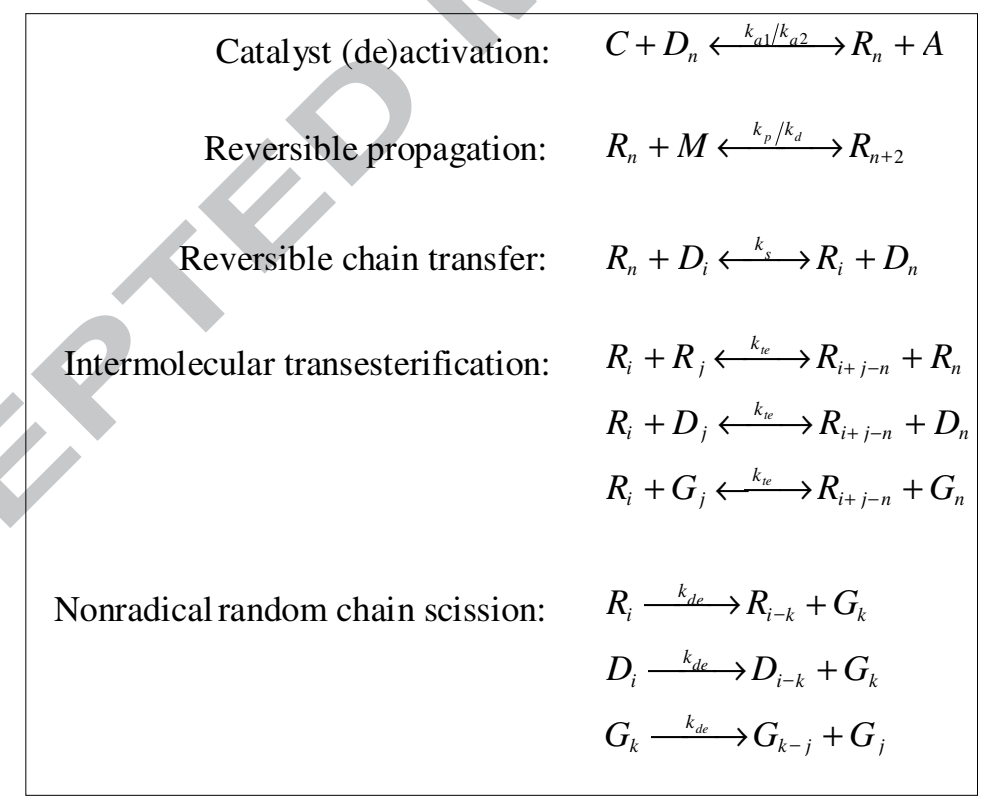

Figure 3. Kinetic scheme of L,L-lactide ROP according to [21].

The model equations are represented by material balances of monomer, catalyst, octanoic acid, and population balances of polymer moments of zero, first and second order for all three types of chains. System 
of 12 ordinary differential equations (ODEs) is then integrated by convenient solver for stiff problems, in our case Fortran adaptation of ODEPACK [56].

Given the value of initial concentration of monomer in the reaction mixture, there are 14 independent input parameters of the model. Parameters of the reaction recipe involve reaction temperature $T$ (isothermal reactor is assumed), initial concentration of catalyst, co-catalyst and octanoic acid (which acts as an impurity) in the system denoted as $\left[C_{0}\right],\left[D_{0}\right]$ and $\left[A_{0}\right]$, respectively.

Kinetic parameters have been estimated from experimental data [21] and include: rate coefficient of catalyst activation and chain transfer, $k_{a 1}$ and $k_{s}$, resp. (independent of temperature), preexponential factor of propagation, intermolecular transesterification and chain scission, $k_{p 0}, k_{t e 0}, k_{d e 0}$, resp., activation energy of propagation, intermolecular transesterification and chain scission, $E_{a, p}, E_{a, t e}, E_{a, d e}$. Note that the catalyst deactivation rate $k_{a 2}$ is evaluated as $k_{a 2}=k_{a 1} / K_{e q, a}$, where activation equilibrium constant $K_{e q, a}$ is assumed to be dependent on the temperature. By regressing the $\ln \left(K_{e q, a}\right)$ versus $1 / T$ (given in $\mathrm{K}^{-1}$ ) measured dependency taken from [21] we arrived at the following expression for $K_{\text {eq,a }}$ (more information about the regression can be found in Supplementary Information) :

$$
K_{e q, a}=\exp \left(\frac{-6029.3}{T}+11.943\right)
$$

Additionally, value of depropagation rate coefficient $k_{d}$ is evaluated as $k_{d}=k_{p}\left[M_{e q}\right]$. Temperature dependency of the monomer equilibrium constant $\left[M_{e q}\right]$ introduces two additional fundamental input parameters not mentioned in [21]: heat and entropy of the polymerization, $\Delta H_{l c}, \Delta S_{l c}$, respectively, via [57]

$$
\left[M_{e q}\right]=\exp \left(\frac{\Delta H_{l c}}{R T}-\frac{\Delta S_{l c}}{R}\right)
$$

\subsection{Stochastic (Monte Carlo) Simulation}

Backbone of the MC simulation of L,L-lactide batch ROP is based on the well-known stochastic simulation algorithm (SSA) for dynamic modeling of coupled chemical reactions [55], which became extremely popular 
in the polymer reaction engineering and initiated extensive use of Monte Carlo models in the field $[58,59,60,61,62]$. The algorithm can be briefly summarized in the following steps:

(1) The initial macroscopic concentrations of chemical species in the reaction mixture are converted to the microscopic (i.e., number concentrations) via Avogadro number $N_{A}$ and the simulated volume $V$, which is related to the chosen number of simulated "particles" (molecules) $N_{p}$ (i.e., size of the statistical ensemble). The same is carried out for macroscopic deterministic kinetic rate coefficients $k^{d e t}$, which are transformed into microscopic rate coefficients $k^{M C}$. Simulation time is set to zero.

(2) In each iteration of the MC simulation, rates of elementary reaction steps are updated based on kinetic rates $k^{M C}$ and current concentrations of species. Probability $p_{i}$ of $i$-th reaction step is then evaluated as

$$
p_{i}=\frac{R_{i}}{\sum_{j=1}^{M} R_{j}},
$$

where $R_{i}$ is the rate of $i$-th elementary reaction step and $M$ is the total number of assumed elementary reaction steps.

(3) The integer index $j$ of the reaction to happen $(1 \leq j \leq M)$ is randomly selected if the random number $r_{1}$ from the uniform distribution and interval $[0,1]$ lies in the range

$$
\sum_{i=1}^{j-1} p_{i}<r_{1} \leq \sum_{i=1}^{j} p_{i}
$$

(4) The reaction selected using Eq. (4) is implemented, i.e., concentrations of the corresponding species and lengths of polymer chains are updated accordingly.

(5) The simulation time increment $\Delta t$ is estimated using second random number $r_{2}$ uniformly distributed from interval $[0,1]$ :

$$
\Delta t=\frac{-\ln r_{2}}{\sum_{i=1}^{M} R_{i}} .
$$

Based on the increment $\Delta t$, the current simulation time is updated.

(6) Steps (2)-(5) are repeated until the desired time or conversion is achieved. 
In order to enhance the computational efficiency, hybrid methods are sometimes introduced, successfully combining stochastic (MC) calculations with solutions provided by deterministic (ODE) models. Recent examples of problems treated by such hybrid MC approach include for example distribution of functional groups in copolymerization [63], modeling of low-density polyethylene (LDPE) production in high pressure tubular and autoclave reactors [64], or production of methyl methacrylate (MMA) by free radical polymerization [65]. Here we develop our own hybrid model suitable for lactide ROP, based on the following assumptions:

- In the range of experimental conditions investigated here, the equilibrium between active and dormant chains is achieved almost instantaneously after the beginning of the reaction. Therefore their concentration during the reaction is considered constant during the process and the catalyst activation/deactivation reaction steps are not considered in MC simulation. Total number of assumed reaction steps $M$ is thus equal to 9.

- Before the MC simulation is initiated, dynamic evolution of elementary reaction rates calculated by the macro-scale simulation is stored and converted into microscopic (MC) format (see Table 1). In each iteration of MC algorithm, values of reaction rates at current simulation time are extracted via linear interpolation between two neighboring points (in time series) generated by macro-scale model.

The hybrid MC simulation thus relies heavily on data pre-calculated by macroscopic model and represents its extension, carrying out mainly topological operations with polymer chains. In this respect we derive benefit from the living behavior of the lactide ring opening polymerization (i.e., constant concentration of active and dormant chains).

Reaction rate functions $R_{i}^{M C}(t)$ used to interpolate the actual reaction rates in each MC iteration, are presented in Table 1. Let us now explain relation between the deterministic rate coefficients used in the macro-scale model (denoted by superscript "det") and their microscopic counterparts (with 
superscript "MC"). For bi-molecular reactions (propagation, chain transfer, transesterification), the relation is as follows,

$$
k_{i}^{M C}=k_{i}^{\text {det }} /\left(N_{A} V\right), \quad i=p, s, t e,
$$

while in the case of unimolecular reactions (depropagation, chain scission), the values of macroscopic and microscopic kinetic rates are equal:

$$
k_{i}^{M C}=k_{i}^{d e t}, \quad i=d, d e .
$$

The rate functions in Table 1 depend on the dynamic profiles of monomer concentration $[M]$ and (zero and first order) moments of active, dormant and terminated chains $\lambda, \mu, \gamma$, respectively. All these quantities are accessible from the macro-scale simulation (as $X^{\text {det }}$ ) and converted to their dimensionless microscopic counterparts $\left(X^{M C}\right)$ according to

$$
X^{M C}=X^{d e t} N_{A} V, \quad X=[M], \lambda_{0}, \lambda_{1}, \mu_{0}, \mu_{1}, \gamma_{0}, \gamma_{1} .
$$

Following the definition of polymer moments, concentration of active and dormant chains is represented by zero order moments of the corresponding chains $\lambda_{0}$ and $\mu_{0}$, respectively. Since their values are considered constant during MC simulation, only the final values from the macro-scale simulation $\lambda_{0, f}, \mu_{0, f}$ are used in the rate functions. It is evident from Table 1 that the rates of depropagation and of chain transfer are actually constant and can be therefore evaluated before the iterative part of the MC simulation starts.

Table 1. Functions representing rates of elementary reaction steps implemented in the hybrid Monte Carlo simulation.

\begin{tabular}{lll}
\hline Index & Reaction step & Corresponding rate function \\
\hline 1 & $R_{n}+M \stackrel{k_{p}}{\longrightarrow} R_{n+2}$ & $R_{p}^{M C}(t)=k_{p}^{M C}[M]^{M C}(t) \lambda_{0, f}^{M C}$ \\
2 & $R_{n} \stackrel{k_{d}}{\longrightarrow} R_{n-2}+M$ & $R_{d}^{M C}=k_{d}^{M C} \lambda_{0, f}^{M C}$ \\
3 & $R_{n}+D_{i} \stackrel{k_{s}}{\longrightarrow} R_{i}+D_{n}$ & $R_{s}^{M C}=2 k_{s}^{M C} \lambda_{0, f}^{M C} \mu_{0, f}^{M C}$ \\
4 & $R_{i}+R_{j} \stackrel{k_{t e}}{\longrightarrow} R_{i+n}+R_{j-n}$ & $R_{t e R}^{M C}(t)=k_{t e}^{M C}\left(\lambda_{1}^{M C}(t)-\lambda_{0, f}^{M C}\right) \lambda_{0, f}^{M C}$
\end{tabular}




$$
\begin{array}{lll}
5 & R_{i}+D_{j} \stackrel{k_{t e}}{\longrightarrow} R_{i+n}+D_{j-n} & R_{t e D}^{M C}(t)=k_{t e}^{M C}\left(\mu_{1}^{M C}(t)-\mu_{0, f}^{M C}\right) \lambda_{0, f}^{M C} \\
6 & R_{i}+G_{j} \stackrel{k_{t e}}{\longrightarrow} R_{i+n}+G_{j-n} & R_{t e G}^{M C}(t)=k_{t e}^{M C}\left(\gamma_{1}^{M C}(t)-\gamma_{0}^{M C}(t)\right) \lambda_{0, f}^{M C} \\
7 & R_{i} \stackrel{k_{d e}}{\longrightarrow} R_{i-k}+G_{k} & R_{d e R}^{M C}(t)=k_{d e}^{M C}\left(\lambda_{1}^{M C}(t)-\lambda_{0, f}^{M C}\right) \\
8 & D_{i} \stackrel{k_{d e}}{\longrightarrow} D_{i-k}+G_{k} & R_{d e D}^{M C}(t)=k_{d e}^{M C}\left(\mu_{1}^{M C}(t)-\mu_{0, f}^{M C}\right) \\
9 & G_{k} \stackrel{k_{d e}}{\longrightarrow} G_{k-j}+G_{j} & R_{d e G}^{M C}(t)=k_{d e}^{M C}\left(\gamma_{1}^{M C}(t)-\gamma_{0}^{M C}(t)\right)
\end{array}
$$

The chain lengths are stored in arrays $(R, D, G)$ with amount of elements in the array representing number of the respective type of chains (active, dormant, terminated) and the value stored in each array element denoting the chain length (i.e., degree of polymerization) of a given chain. Based on the number of chains assumed in the statistical ensemble $N_{p}$, the simulated volume $V$ is estimated with the aim of Avogadro number and final values of active, dormant and terminated chain concentrations (taken from the macro-scale simulation) as follows

$$
V=\frac{N_{p}}{N_{A}\left(\lambda_{0, f}^{d e t}+\mu_{0, f}^{d e t}+\gamma_{0, f}^{d e t}\right)}
$$

At the beginning of the simulation $(t=0)$, the number of terminated chains is set to zero, while the ratio of active and dormant chains follows the (constant) value predicted by the macro-scale simulation. Also, the degree of polymerization for both active and dormant chains is set to 1 before the simulation is initiated.

After the reaction step (cf. Table 1) is selected based on Eq. (4), it is implemented, i.e., the chain-length distribution is updated. In the case of propagation and depropagation, this step is straightforward: the index of an active chain is randomly selected and its chain length is increased (or decreased) by 2 . For the chain transfer step (index 3 in Table 1), both active and dormant chains are selected randomly and their chain lengths are simply interchanged. If the intermolecular transesterification is selected (index 4-6), the situation is a bit more complicated. In the case of reaction of two active chains (index 4), the two different chains are selected randomly and a portion (i.e., number of repeating units) of the attacked chain is randomly selected, subtracted from it, and added to the attacking chain. When two different types of 
chain react (index 5-6), their selection procedure is different: the active chain (always the attacking one) is selected randomly, while the probability of selecting the attacked chain (dormant or terminated) is proportional to its chain length. This can be easily implemented via following condition [29]

$$
D P_{\text {select }} \geq r n d \times \max (\text { dist })
$$

where $D P_{\text {select }}$ represents the degree of polymerization of the selected chain, $r n d$ is a random number from uniform distribution between 0 and 1 , and $\max (d i s t)$ denotes the maximum chain length achieved so far in the distribution of the attacked chains. Originally, we did not use Eq. (10) for selecting the attacked chain during transesterification step. Instead, we used our own procedure which involved generation of normalized cumulative chain length distribution and a uniformly distributed random number from the interval $[0,1]$ which was used to inversely obtain the selected chain length from the distribution. In this way higher probability of longer chains being selected can be naturally implemented. However, later we found Eq. (10) in the recent paper [29] and decided to test it in our model. Although we were not able to find or derive any theoretical support for its use, Eq. (10) allowed us to produce the same results as our own method, but in shorter computational time. Therefore we decided to keep the equation in the model, even though we see its use as a rather empirical approach to the problem. The condition expressed by Eq. (10) is also applied during selection of chain undergoing nonradical scission (index 7-9) with the length of a newly generated terminated chain being selected randomly from the original chain length.

The values of number and weight average molar masses of the actual MMD $M_{n}$ and $M_{w}$ can be evaluated during the $\mathrm{MC}$ simulation as

$$
\begin{aligned}
& M_{n}=M_{M} \frac{\sum_{i=1}^{N_{R}} D P(i)+\sum_{j=1}^{N_{D}} D P(j)+\sum_{k=1}^{N_{G}} D P(k)}{N_{R}+N_{D}+N_{G}}, \\
& M_{w}=M_{M} \frac{\sum_{i=1}^{N_{R}} D P(i)^{2}+\sum_{j=1}^{N_{D}} D P(j)^{2}+\sum_{k=1}^{N_{G}} D P(k)^{2}}{\sum_{i=1}^{N_{R}} D P(i)+\sum_{j=1}^{N_{D}} D P(j)+\sum_{k=1}^{N_{G}} D P(k)},
\end{aligned}
$$

where $M_{M}$ denotes the molar mass of monomer (lactoyl) unit, $N_{R}, N_{D}, N_{G}$ represent total number of active, dormant and terminated chains, respectively, and $D P(i)$ stands for the length of the $i$-th chain. 


\subsection{Prediction of Rheological Properties}

In addition to the prediction of full molar mass distribution of the produced PLLA by means of the hybrid Monte Carlo simulation, we use the predicted polymer molecular architecture to estimate its rheological properties in a molten state. By doing so, we (i) extend the palette of the product properties predicted by those important especially in polymer down-stream processing, and at the same time (ii) get a possibility of validating the developed modeling framework by experimental data on PLLA rheology published in the literature.

Flow properties of viscoelastic polymer melts are usually experimentally characterized by the so-called frequency sweep measurements, in which the sample is deformed in an oscillatory way with constant amplitude and varying frequency $\omega$. Output of such experiments is then represented by the frequency dependencies of storage modulus $G^{\prime}(\omega)$, and loss modulus $G^{\prime}(\omega)$, which represent elastic and viscous parts of the material undergoing deformation [66]. The storage and loss moduli can be also combined into the complex viscosity defined as [67]

$$
\eta^{*}(\omega)=\sqrt{\left(\frac{G^{\prime}(\omega)}{\omega}\right)^{2}+\left(\frac{G^{\prime \prime}(\omega)}{\omega}\right)^{2}} .
$$

The above mentioned rheological characteristics of polymer melts depend on many factors including temperature, polymer density and molecular architecture (molar mass distribution, branching, cross-linking, chain entanglement, etc.).

When assessing the frequency dependency of storage and loss moduli, several important characteristics can be defined. For instance, crossover modulus $G_{c}$, i.e., point in frequency sweep plot where storage and loss moduli have equal value $G^{\prime}(\omega)=G^{\prime}(\omega)$, is sensitive to changes in polymer chain-length distribution $[68,69,70]$ : horizontal position of $G_{c}$ point in frequency sweep plot is sensitive to average chain length (or average molar mass), while its vertical position is associated with broadness of the chain-length distribution (or MMD). Another important rheological characteristics of polymer melts is the so-called 
plateau modulus $G_{N}^{0}$, which is proportional to the intensity of chain entanglement [71] and can be estimated as the value of storage modulus where loss modulus $G^{\prime \prime}(\omega)$ exhibits local minimum and at the same time storage modulus $G^{\prime}(\omega)$ has an inflection point.

Various correlations for estimation of PLA melts complex viscosity have been published [30], but we use the "BoB" software for prediction of rheological properties of homopolymers with general branch-on-branch architecture. This can be viewed as a redundant work since here we present study of only linear (non-branched) PLLA. However, it is our plan to add chain branching (by means of multifunctional co-catalysts, e.g. diols) to the developed model in the future in order to allow for efficient in silico design of PLA-based materials with general molecular architecture and targeted rheological properties.

"BoB" [51] represents state-of-the-art software tool for prediction of rheological behavior of branched polymers with general architecture, based on the tube theory of polymer chain entanglements $[72,73]$. BoB is a public domain software ${ }^{1}$, which has been employed notably in a pioneering study connecting molecular topology of low-density polyethylene (LDPE) samples of industrial-level complexity with their rheological behavior $[74,75]$.

In our framework, we use the latest version of $\mathrm{BoB} 2.5$. To perform calculations, it needs various input data. First, the molecular architecture of the polymer must be provided in a convenient form (for more details, see documentation of $\mathrm{BoB} 2.3$ release). For the memory allocation, information about the maximum number of input polymer molecules $N_{p}$ and their segments $\left(2 N_{p}\right.$ for linear chains) must be provided. Physicochemical input parameters include dynamic dilation exponent $\alpha$, molar mass of monomer unit $M_{M}$, and temperature $T$ and density $\rho$ of polymer melt. Last but not least, two parameters need to be estimated from the experimental data: the number of monomer units in one entangled segment $N_{e}$, and the entanglement time $\tau_{e}$, which represents relaxation time of the chain segment between two entanglement points. For the readers interested in the BoB software, simple example of its usage is available in Supplementary Information.

\footnotetext{
${ }^{1}$ https://sourceforge.net/projects/bob-rheology/files/bob-rheology/
} 
$G^{\prime}(\omega), G^{\prime}(\omega)$ dependencies are provided by BoB in the form of table with discrete values of moduli over a wide range of frequency values (implicit range is $10^{-6}-10^{8} \mathrm{~s}^{-1}$, but this can be modified by the user) so the automatic evaluation of crossover and plateau moduli $G_{c}, G_{N}^{0}$, resp., had to be implemented. There are various approaches for evaluation of plateau modulus from frequency sweep plot [76]; we applied approach suggested by $\mathrm{Wu}$ [77], in which the plateau modulus is found as the value of storage modulus $G^{\prime}$ at minimum of $\tan (\delta)=G^{\prime} / G^{\prime}$ ratio:

$$
G_{N}^{0}=\left.G^{\prime}(\omega)\right|_{\tan \delta \rightarrow \min }
$$

At the cross over of both moduli, the value of $\tan (\delta)=G^{\prime} \prime / G^{\prime}$, should be equal to 1 . Therefore the crossover modulus can be found as a minimum on the $|\tan (\delta)-1|$ dependency:

$$
G_{c}=\left.G^{\prime}(\omega)\right|_{|\tan \delta-1| \rightarrow \min }=\left.G^{\prime \prime}(\omega)\right|_{|\tan \delta-1| \rightarrow \min }
$$

Illustration of localization of plateau and crossover moduli in the frequency sweep plot predicted by BoB is presented in Figure 4. Validity of Eqs. (14) and (15) has been successfully tested over several virtually generated polymer samples with wide range of MMD properties.

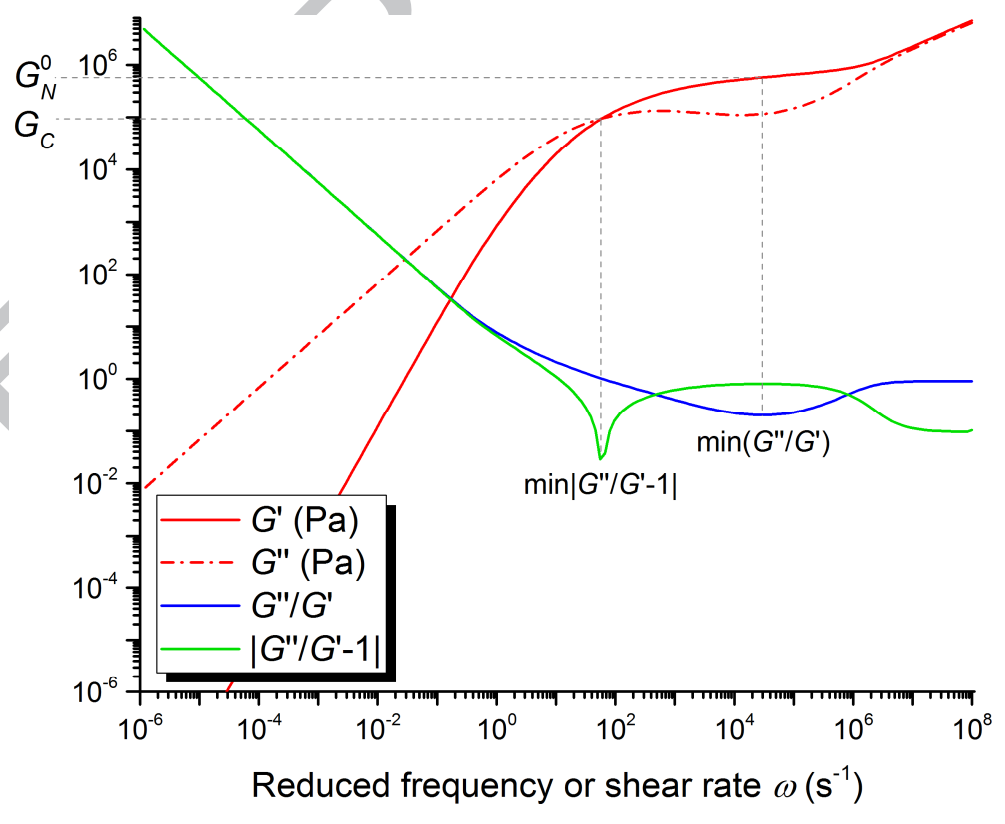

Figure 4. Illustration of localization of crossover and plateau moduli $G_{c}, G_{N}^{0}$, respectively, in frequency sweep data predicted by BoB software. 


\subsection{Sensitivity Analysis}

In order to perform the parametric sensitivity analysis of the model presented above, one can start with the most widely used local methods, which are also called "one factor at a time" (OAT) methods. In this approach, each input parameter of a model is varied (i.e., perturbed) one at a time around its nominal value, and the resulting effect on the output is measured. The local sensitivity measures are typically defined using the first order derivative of a model output $y=f(x)$, with respect to an input parameter $x$ [78]:

$$
\begin{aligned}
& \text { absolute sensitivity: } s_{a}=\frac{\partial y}{\partial x}, \\
& \text { relative sensitivity: } s_{r}=\frac{\partial y}{\partial x} \frac{x_{0}}{y_{0}},
\end{aligned}
$$

where $x_{0}$ and $y_{0}$ represent the nominal value of model parameter and the corresponding model output $y_{0}=$ $f\left(x_{0}\right)$, respectively. The relative sensitivity functions are non-dimensional with respect to units and are commonly used to compare the effects of model inputs among each other. The derivatives can be evaluated either analytically or numerically (using finite difference formulas), depending on the model complexity.

Results of local sensitivity analysis are valid only in close proximity to the parameters analyzed and depend on their nominal values. The alternative possibility is to use regional or global methods, which expand the analysis from one point in the parameter space to a broader range, however at the expense of significantly higher computational cost. Here we are going to explore two of these methods: (i) the elementary effects method, and (ii) the variance-based method of Sobol.

The elementary effects method (also known as Morris screening) is relatively simple and computationally effective approach for fast screening of input parameters importance on the model output [79]. Assuming model with $k$ independent inputs $x_{i}, i=1 \ldots k$, its input space represented by the $k$ dimensional unit cube is discretized into $p$-level grid $\boldsymbol{\Omega}$. For a given value of input vector $\mathbf{x}$, the elementary effect of the $i$-th input parameter is defined as 


$$
E E_{i}=\frac{y\left(x_{1}, x_{2}, \ldots, x_{i-1}, x_{i}+\Delta, \ldots, x_{k}\right)-y\left(x_{1}, x_{2}, \ldots, x_{k}\right)}{\Delta}
$$

where symbol $\Delta$ denotes the perturbation step, which is a function of $p$, and $y$ is the scalar model output. Eq. (18) remarkably resembles the well-known forward difference formula and the Morris method can thus be characterized as an approach applying local sensitivity analysis in a global context by means of sophisticated sampling method. The sampling of the input parametric domain $\Omega$ is carried out $r$ times: each sampling run starts by random selection of a point $\mathbf{x}^{*}$ in the $p$-level grid of $\Omega$ and the subsequent point on the sampling trajectory is generated by increasing one or more components of $\mathbf{x}^{*}$. The subsequent points differ from the previous only in one component $i$, which has been either increased or decreased by $\Delta$. Each sampling procedure results in a trajectory of $(k+1)$ points, therefore requiring $r(k+1)$ model evaluations in total to obtain necessary model output for all input samples. Finally, the distribution of elementary effects among different sampling trajectories is assessed, providing three important measures of the $i$-th input parameter sensitivity:

$$
\begin{aligned}
& \mu_{i}=\frac{1}{r} \sum_{j=1}^{r} E E_{i}^{j}, \\
& \mu_{i}^{*}=\frac{1}{r} \sum_{j=1}^{r}\left|E E_{i}^{j}\right|, \\
& \sigma_{i}^{2}=\frac{1}{r-1} \sum_{j=1}^{r}\left(E E_{i}^{j}-\mu_{i}\right)^{2} .
\end{aligned}
$$

Both computational cost and accuracy of the Morris method depend on the values of $p, \Delta$ and $r$. For an even value of $p$, Morris recommends $\Delta=p /[(2(p-1)]$. Value of $r$ should be proportional to $p$ : it has been demonstrated that the choice of $p=4$ and $r=10$ produced valuable results [80,81]. These parameter settings were also applied in our study, results of which are presented in Section 3.3.

Another important class of global sensitivity analysis methods is aiming at measuring how variation in a given model input parameter contributes to variance in the model output. Among these variance-based methods, the state of the art is represented by the Monte Carlo method of Sobol [82], later extended by Saltelli and co-workers $[83,84]$. 
Having a mathematical model with $k$ input parameters, the method starts by generating a $(N \times$ $2 k$ ) matrix of random numbers from the input parametric space. $N$ is called a base sample, typically on the order of hundreds or thousands. The matrix is then divided into two matrices $\mathbf{A}$ and $\mathbf{B}$, each of them containing half of the sample. For $i$-th input parameter investigated, matrix $\mathbf{A}_{\mathbf{B}}^{i}$ (alternatively $\mathbf{B}_{\mathbf{A}}^{i}$ ) is created, which is formed by all columns (representing individual parameters) of $\mathbf{A}$ except the $i$-th column, which is taken from matrix $\mathbf{B}$. For all samples from the input space (i.e., for all rows of matrices $\mathbf{A}, \mathbf{B}$, and $\mathbf{A}_{\mathbf{B}}^{i}$ ) the model output is evaluated, resulting in three vectors of length $N: y(\mathbf{A}), y(\mathbf{B})$ and $y\left(\mathbf{A}_{\mathbf{B}}^{i}\right)$. In order to investigate all $k$ parameters, the method thus requires $N+N+k N=N(k+2)$ model evaluations, which is much more efficient than $N^{2}$ runs needed by the brute-force-method [36]. Three output vectors are finally used to calculate the two commonly used measures of parameter importance: (i) first order sensitivity index $S_{i}$, representing the dominant (first order) contribution of the $i$-th input parameter to the variance of the output, and (ii) total sensitivity index $S_{T i}$, which accounts for the total contribution to the output variation due to the $i$-th input, i.e., its first plus higher order effects due to interactions with other inputs. There are various approaches for evaluation of the sensitivity indices, Saltelli et al. [85] recommend the following expressions originally proposed by Jansen [86]:

$$
\begin{aligned}
& S_{i}=1-\frac{1 /(2 N) \sum_{j=1}^{N}\left(y(\mathbf{B})_{j}-y\left(\mathbf{A}_{\mathbf{B}}^{i}\right)_{j}\right)^{2}}{V(y)}, \\
& S_{T_{i}}=\frac{1 /(2 N) \sum_{j=1}^{N}\left(y(\mathbf{A})_{j}-y\left(\mathbf{A}_{\mathbf{B}}^{i}\right)_{j}\right)^{2}}{V(y)},
\end{aligned}
$$

where symbol $V(y)$ represents variance of the model output,

$$
V(y)=1 / N \sum_{j=1}^{N}\left(y(\mathbf{A})_{j}\right)^{2}-\left(\frac{1}{N} \sum_{j=1}^{N} y(\mathbf{A})_{j}\right)^{2} .
$$

In Eqs. (22) and (23), subscript $j$ denotes the $j$-th element of the output vectors. Thanks to the output variance occurring in denominators, both indices are normalized, so that

$$
0 \leq S_{i} \leq 1, \quad \wedge \sum_{i=1}^{k} S_{i} \leq 1, \wedge \sum_{i=1}^{k} S_{T_{i}} \geq 1
$$


It has been demonstrated that the mean of the absolute values of the elementary effects $\mu_{i}^{*}$, cf. Eq. (20), is a reasonable proxy of the total sensitivity index $S_{T_{i}}$ [87]. For more information on the global sensitivity analysis techniques we refer to the textbook by Saltelli et al. [36].

In our study we employed Sobol's method based on Monte Carlo sampling of the input space, in which the input samples were generated as scrambled quasi-random Sobol sequences $[88,89]$ with uniform distribution. First order and total sensitivity indices were evaluated using Eqs. (22) and (23).

\section{Results and Discussion}

\subsection{Model Validation}

A natural way to validate the developed model would be to compare its predictions of polymer molar mass distribution (MMD) and rheological characteristics with gel permeation (size exclusion) chromatography and frequency sweep data measured experimentally for various PLLA samples. However, due to the lack of experimental facilities we have to rely purely on data from the literature. Since we were not able to find any study that would involve both full MMD and rheological characterization of PLLA samples, we decided to use data from the well-known paper by Cooper-White and Mackay [31] in which three commercial PLLA samples (Resomers L206, L210, L214) produced by Boehringer Ingelheim are characterized by frequency sweep measurements and accompanied by information about their weight average molar mass $M_{w}$ and polydispersity index $P D I=M_{w} / M_{n}$. Our approach to the model validation can be summarized in two steps:

1. By manipulating the reaction conditions, namely temperature $T$, relative monomer and co-catalyst concentrations $\left[M_{0}\right] /\left[C_{0}\right],\left[D_{0}\right] /\left[C_{0}\right]$, respectively, we use the macro-scale model to in silico generate polymer samples with $M_{w}$ and $P D I$ as close as possible to the commercial samples.

2. Using BoB software, we evaluate the frequency dependencies of storage and loss moduli $G^{\prime}(\omega)$, $G^{\prime}$ ' $(\omega)$, respectively, for the artificially generated samples and compare them with those measured 
[31]. For the rheological prediction, two input parameters need to be estimated: number of monomer units in one entangled segment $N_{e}$, and the entanglement time $\tau_{e}$.

The selected validation approach has two main limitations. First, the micro-scale model cannot be directly validated by experimental data, i.e. by comparing predicted and measured full molar mass distribution (MMD) of the produced polymer. In our approach, the micro-scale model is validated only indirectly via (i) its influence on the meso-scale (rheological) prediction, and by (ii) comparison of the averaged MMD characteristics $\left(M_{n}, M_{w}\right)$ with those provided by macro-scale model. Second, having a possibility of lab-scale polymer synthesis of samples L206, L210 and L214 in vitro would very probably result in a revision (i.e. reestimation) of some model parameters. In this context, the reaction conditions leading to in silico production of the samples presented in Table 2 have somewhat limited validity, unless they are verified by real experiment.

Mass-average molar mass $M_{w}^{e x p}$ and polydispersity $P D I^{e x p}$ of samples L206, L210 and L214 published by Cooper-White and Mackay [31] are listed in Table 2 along with $M_{w}{ }^{s i m}$ and $P D I^{\text {sim }}$ of the samples generated in silico and model parameters manipulated to achieve the desired values. Besides the above mentioned model input parameters which affect the reaction conditions, other parameters of the macro-scale model retained their nominal values (cf. Table 3). Reaction time in all simulations was set to 12 min, corresponding to monomer conversion around $95 \%$. Common inputs to the rheological property prediction performed by BоB were: dynamic dilation exponent $\alpha=1.0$, molar mass of monomer (lactoyl) unit $M_{M}=$ $72.06 \mathrm{~g} / \mathrm{mol}$, temperature of the polymer melt (i.e., sample temperature during the frequency sweep measurement) $T_{m}=200^{\circ} \mathrm{C}$ and density of the polymer melt $\rho_{m}=1.090 \mathrm{~g} / \mathrm{cm}^{3}\left(T_{m}\right.$ and $\rho_{m}$ taken from [31]). Broadly speaking, BoB inputs primarily determining the predicted rheological characteristics are polymer chain architecture (provided by micro-scale model), and two estimated parameters: average number of monomer units in one entangled segment $N_{e}$, and the entanglement time $\tau_{e}$. Effect of other BoB inputs on the output is studied in Section 3.3. 

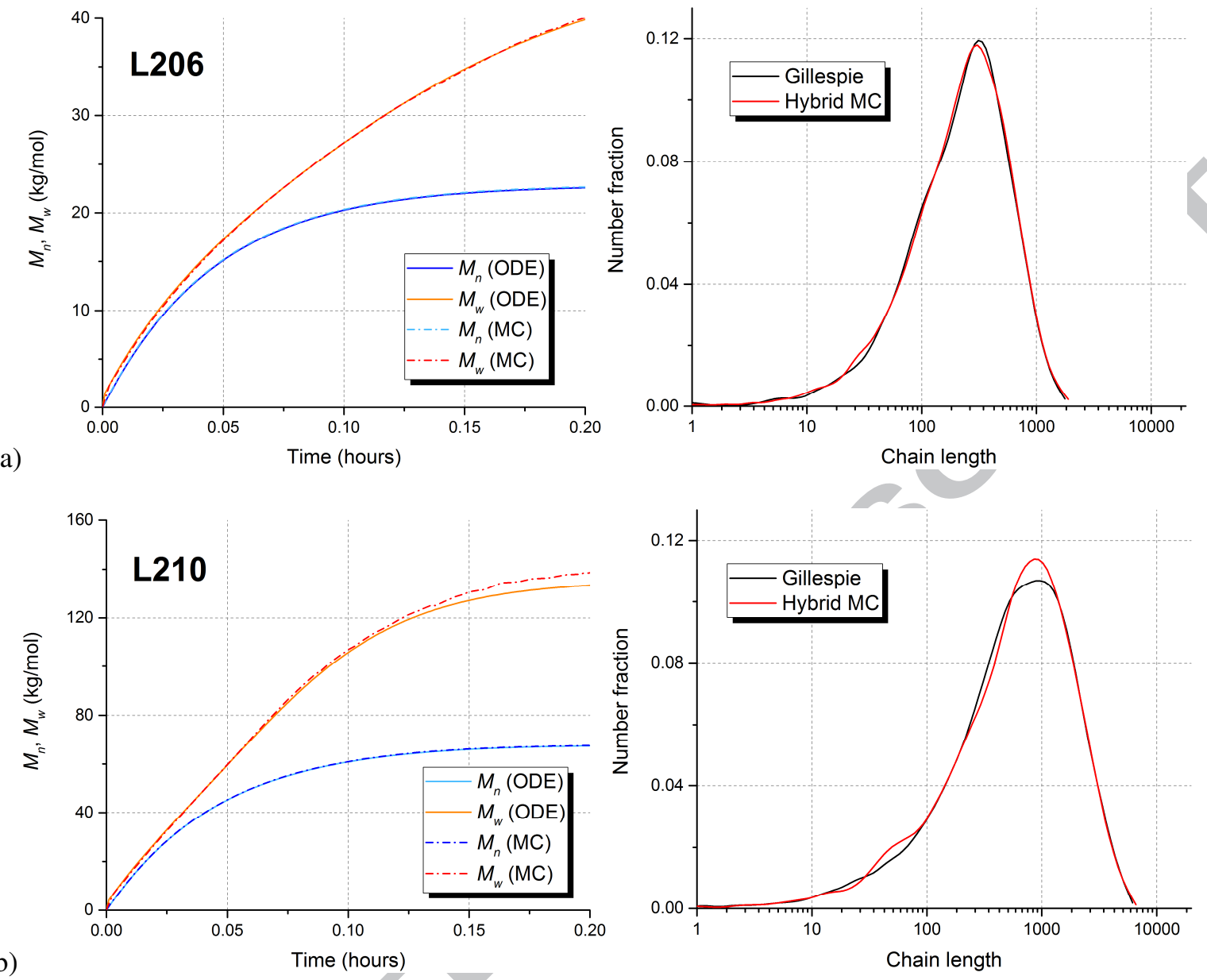

(b)
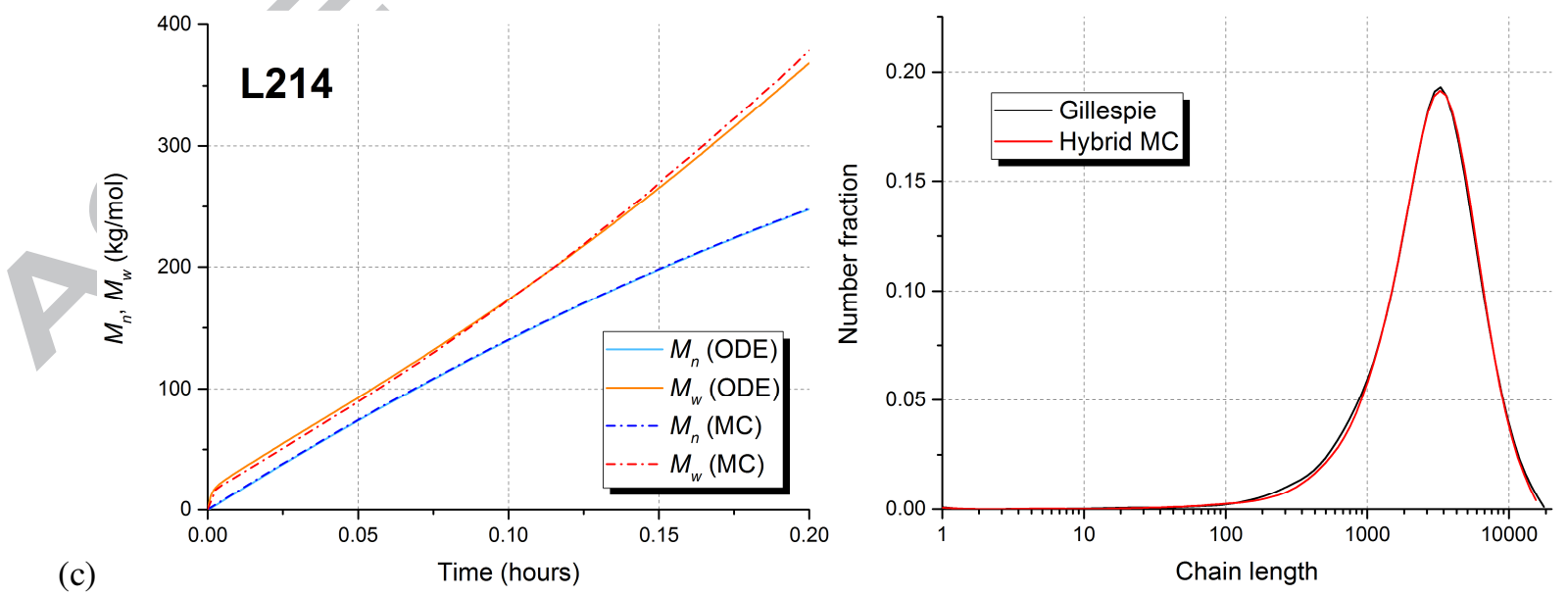
Figure 5. Results of simulated production of samples (a) L206, (b) L210 and (c) L214 (cf. Table 2) by L,L-lactide ring opening polymerization. Left: Comparison of macro-scale model (ODE) and Monte Carlo (MC) predictions of dynamic evolution of number and weight average molar masses $M_{n}, M_{w}$, resp. Right: Final number chain length distribution (NCLD) predicted by traditional SSA algorithm by Gillespie and the Hybrid MC method presented in this paper.

Comparison of outputs provided by macro-scale (ODE-based) and micro-scale (hybrid MC) simulation is an important step in the model validation and internal consistency check. Figure 5 (left) illustrates very good agreement in the dynamic evolution of the number and weight average molar masses $M_{n}, M_{w}$, for samples L206, L210 and 214 predicted by both models with only small deviations in $M_{w}$ towards the end of the reaction. Generally, profiles of mass-average molar mass $M_{w}$ calculated by MC simulation exhibit slightly higher deviations than those of $M_{n}$ due to the intensive "chain re-shuffling" (transesterification) and degradation (scission) reactions involved. In Section 3.2, we will thus use the deviations in $M_{w}$ between ODE and $\mathrm{MC}$ simulation as a measure of the MC simulation accuracy. Another important validation of the developed hybrid MC model is its benchmarking against the traditional SSA algorithm of Gillespie via comparison of full chain length distribution (CLD) predicted by both methods. Figure 5 (right) illustrates that the agreement in calculated final CLD is very good for all three samples, although the number of molecules $N_{p}$ involved in the SSA-based simulation is significantly higher than in hybrid MC model in order to achieve similar numerical accuracy of both models (see further discussion in Section 3.2).

Figures 6-7 show comparison of predicted and experimental values (taken from [31]) of storage modulus $G^{\prime}$, loss modulus $G^{\prime}$, and complex viscosity $\eta^{*}$ for all studied polymer melts at $200^{\circ} \mathrm{C}$ (displayed frequency range follows the experimental data; BoB software allows prediction in significantly higher range). Overall good agreement between simulations and measurements was achieved by assuming value of the entanglement time $\tau_{e}$ to be $9 \times 10^{-7} \mathrm{~s}$ for all samples (values of $\tau_{e}=1.3 \times 10^{-6}$ have been estimated e.g. for linear polybutadienes at room temperature [90]) and molar mass between entanglements $M_{e}=N_{e} M_{M}$ to be in the range $4200-4700 \mathrm{~g} / \mathrm{mol}$, which perfectly corresponds to the approximate value of $M_{e} \sim 4000$ $\mathrm{g} / \mathrm{mol}$ reported for PLA melt at lower temperature $140^{\circ} \mathrm{C}$ [30]. Small discrepancy observed at low 
frequencies for sample L214 can be caused by the difference between properties of the real and artificially generated polymer samples.

Table 2. Comparison of mass-average molar mass $M_{w}$ and polydispersity index $P D I$ of commercial samples studied by Cooper-White and Mackay [31] with model predictions and input parameters.

Property
/Parameter

Figure 6. Comparison of predicted (lines) and measured (points) moduli $G^{\prime}, G^{\prime}$ ' of PLLA samples (cf. Table 2) at $200^{\circ} \mathrm{C}$. Storage modulus $G^{\star}$ is indicated by full lines and points. The experimental data were taken from [31]. 


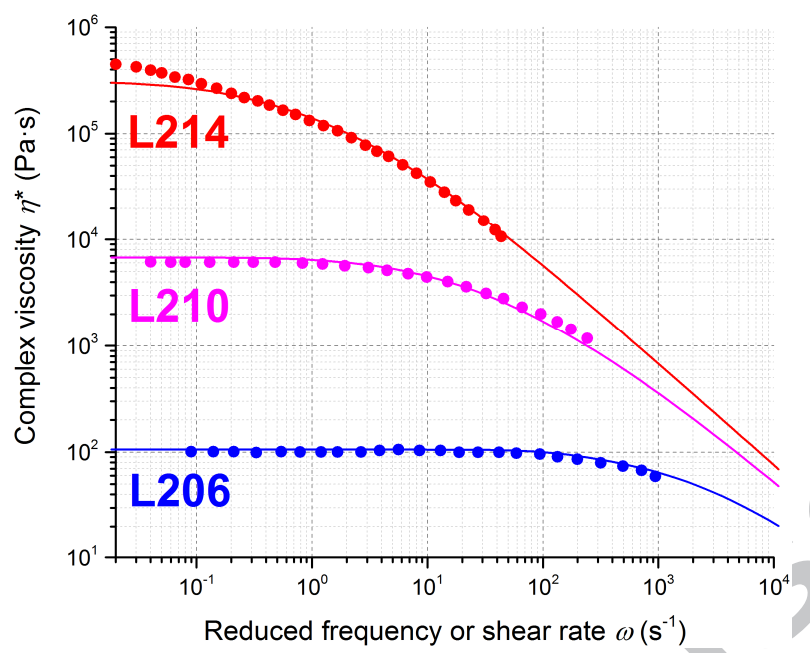

Figure 7. Comparison of predicted (lines) and measured (points) complex viscosity of PLLA samples (cf. Table 2) at $200^{\circ} \mathrm{C}$. The experimental data were taken from [31].

\subsection{Computational Efficiency}

For the model to be useful in optimization (both off-line and on-line) of the polymer production process, its computational efficiency needs to be addressed. Let us analyze the computational load of individual parts of the developed modeling framework: Fortran implementation of (i) the macro-scale (deterministic) part, and of (ii) the micro-scale (stochastic) Monte Carlo simulation, and (iii) rheological prediction by "BoB", called as an executable file from the Fortran code. Subject of all simulations described in this section was the model with nominal set of input parameter values (cf. Table 3) assuming the reaction time of $12 \mathrm{~min}$. The calculations were performed on average office laptop equipped with Intel ${ }^{\circledR} \mathrm{Core}^{\mathrm{TM}}$ i5-5300U CPU $2.30 \mathrm{GHz}$ running on Microsoft Windows ${ }^{\circledR} 7$ 64-bit. No parallelization of the developed codes has been carried out.

The deterministic simulation based on integration of ODEs definitely represents the fastest element of the developed framework. With the constant time step of 3.6 s (i.e., solver ODEPACK is called every $3.6 \mathrm{~s}$ of the reaction time, in total 200 times), the overall duration of the simulation (including saving the results to a file) is $0.5 \mathrm{~s}$. 
In the case of $\mathrm{BoB}$, the simulation time depends primarily on the number of polymer chains considered for the simulation, which can be actually downsampled (i.e., smaller amount of chains is randomly selected) from the population produced by MC simulation. In our calculations (e.g., those leading to the results presented in Figures 6-7), we have not observed any significant improvement of the predicted rheological characteristics if the number of considered chains was increased to values higher than 300 . Therefore this value was used in all simulations, with $\mathrm{BoB}$ running for $2.3 \mathrm{~s}$.

It is not surprising that the bottleneck of the computational time is the Monte Carlo simulation. Due to its stochastic nature, speed of MC algorithm is affected not only by the assumed reaction time but also by the reaction conditions, because algorithmic implementation of the elementary reaction steps varies. For instance, propagation of an active chain by two units is evaluated significantly faster than the attack of an active chain on a dormant chain during intermolecular transesterification. Nonetheless, assuming the reaction duration and conditions to be constant, the highest impact on the time and accuracy of the MC simulation has the number of "particles" (i.e. molecules, chains) considered in the system $N_{p}$. In the original stochastic simulation algorithm (SSA) of Gillespie (described in the beginning of Section 2.2), parameter $N_{p}$ involves all reacting species in the system - in this case molecules of monomer, catalyst, co-catalyst, acid and three distinct types of macromolecules. Gillespie's implementation thus requires $N_{p}$ to be relatively high in order to ensure statistical representation of all species (even those present in very low concentrations) and fails to work if $N_{p}$ is below a critical value represented by the ratio of the highest and lowest concentrations involved in the system chemistry [91]. On the other hand, in our hybrid approach, only the number and topology of the macromolecules is tracked by the MC simulation, therefore allowing for significantly lower values of $N_{p}$ for the same accuracy of the prediction.

Let us compare the simulation time and accuracy of the hybrid $\mathrm{MC}$ algorithm presented in this paper with its alternative implemented as Gillespie's SSA. As a measure of accuracy we take the sum of squared residuals (SSR) for the values of the mass-average molar mass $M_{w}$ predicted by Monte Carlo (MC) and deterministic (ODE) simulation along the process trajectory

$$
\operatorname{SSR}\left(M_{w}\right)=\sum_{i=1}^{P=72}\left(M_{w, i}^{M C}-M_{w, i}^{O D E}\right)^{2}
$$


where $P$ represents the number of prediction points representing the trajectory of $M_{w}(t)$ (output of the simulation was saved every $10 \mathrm{~s}$ of reaction time, arriving finally at $720 \mathrm{~s}=12 \mathrm{~min}$ ). It has been already mentioned that SSR for $M_{w}$ is chosen here because $M_{w}$ exhibits generally higher discrepancy than $M_{n}$ when comparing results of Hybrid MC and deterministic ODE simulation. Thus, for a given value of $\operatorname{SSR}\left(M_{w}\right)$ we believe that value of $\operatorname{SSR}\left(M_{n}\right)$ would be always lower. Figure 8 depicts dependency of both $\operatorname{SSR}\left(M_{w}\right)$ and simulation time on the number of particles $N_{p}$ for both MC implementations (denoted as "Hybrid MC" and "Gillespie"). First of all it should be noted that the minimum $N_{p}$ for Gillespie-based simulation was $10^{4}$ and below this value the algorithm could not fully follow the polymerization kinetics. It is obvious that in either algorithm with increasing $N_{p}$ the precision of the prediction improves at the expense of higher computational time.

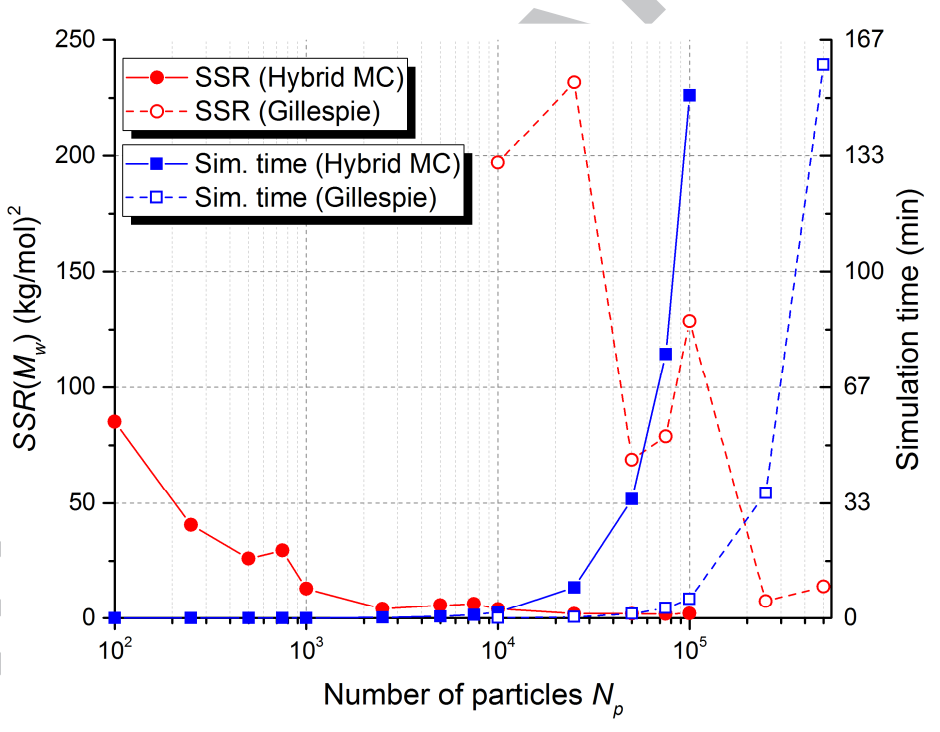

Figure 8. Dependency of sum of squared residuals for the predicted mass-average molar mass $S S R\left(M_{w}\right)$ defined by Eq. (26) and simulation time on the number of particles $N_{p}$ assumed in the original Gillespie Monte Carlo algorithm (denoted as "Gillespie") and the hybrid Monte Carlo ("Hybrid MC") approach developed in this paper.

For the same number of particles $N_{p}$, Gillespie's algorithm performs significantly faster than the hybrid scheme, but with dramatically higher deviation from the accurate results. Both algorithms actually achieve similar precision after very different simulation times: Gillespie's algorithm achieves accuracy of 7.6 
$\mathrm{kg}^{2} / \mathrm{mol}^{2}$ in 35 minutes $\left(N_{p}=2.5 \times 10^{5}\right)$, while hybrid Monte Carlo arrives at $5.7 \mathrm{~kg}^{2} / \mathrm{mol}^{2}$ in 30 seconds $\left(N_{p}=\right.$ $\left.5 \times 10^{3}\right)$, more than 70 times faster.

In this case study, the optimal choice of $N_{p}$ for the hybrid MC approach seems to be between $10^{3}$ and $10^{4}$ (corresponding to the simulation volumes of $2.8 \times 10^{-23}$ and $2.8 \times 10^{-22} \mathrm{~m}^{3}$ ), where the $\operatorname{SSR}\left(M_{w}\right)$ is on the order of units (high accuracy of prediction) and the simulation time below 2 minutes. For example, with $N_{p}=2500$, hybrid MC simulates for $11.4 \mathrm{~s}$ with $\operatorname{SSR}\left(M_{w}\right)=4.14 \mathrm{~kg}^{2} / \mathrm{mol}^{2}$. Using these settings, the prediction of full molar mass distribution and rheological characteristics of the product based on the input reaction conditions (with $94 \%$ monomer conversion achieved) takes less than 15 seconds on a standard PC. Although we should keep in mind that the performance of the micro-scale model will always depend on the actual conditions in the reactor that define the kinetics, these results seem to be promising for the future development of robust soft sensors and model-based systems for control and optimization of L,L-lactide ring opening polymerization reactors.

\subsection{Global Sensitivity Analysis}

In this section we demonstrate application of global sensitivity analysis methods to the modeling framework described in Sections 2.1-2.3. In local (OAT) sensitivity analysis, the model inputs are perturbed only in the close proximity of their nominal values. However, all model parameters have some degree of uncertainty (stemming either from their numerical estimation or from experimental measurement error) that should be taken into account in order to have an overview of model sensitivity in a global context. Therefore we employ two well established methods of regional/global sensitivity analysis, namely Morris screening and Monte Carlo-based method of Sobol (cf. Section 2.4); results of OAT analysis are presented in Supplementary Information.

The model input parameters, their nominal values, uncertainties (i.e. standard deviations for the parameter estimates) and corresponding sampling intervals are presented in Table 3 and divided into 
three groups: (i) reaction conditions, (ii) polymerization kinetic rate coefficients, (iii) and inputs to the BoB software (that have no influence on macro-scale and MC simulation). Uncertainty in the values of species' initial concentrations, rate coefficients $k_{a 1}, k_{s}$ and density of polymer melt $\rho_{m}$ was set to $\pm 5 \%$. The reaction temperature $T$ and temperature of polymer melt $T_{m}$ was assumed to be measured with relatively high precision $( \pm 0.5 \%$ uncertainty). On the other hand, higher uncertainty $( \pm 10 \%)$ was assumed for chain entanglement time $\tau_{e}$ and number of monomer units between entanglements $N_{e}$. Uncertainties of other model parameters was taken directly from [21] and [57] as a result of their numerical estimation. Dynamic dilation exponent $\alpha$ was excluded from the list of uncertain inputs to BoB and its value was set to 1.0 [51,92].

The model outputs of interest consist of the following five process-product characteristics:

1. Conversion of monomer $X$ as an important measure of reaction progress.

2. Number-average molar mass of polymer $M_{n}$ as a direct measure of the average polymer chain length.

3. Polydispersity index $P D I=M_{w} / M_{n}$ is proportional to the broadness (variance) of polymer chain length distribution.

4. Crossover modulus $G_{c}$ is sensitive to changes in polymer chain-length distribution.

5. Plateau modulus $G_{N}^{0}$ as a measure of chain entanglement intensity.

While $X, M_{n}$ and $P D I$ are taken as output of deterministic (macro-scale) simulation, rheological characteristics $G_{c}$ and $G_{N}^{0}$ are predicted by BoB, utilizing polymer chain architecture from stochastic (Monte Carlo) simulation. Therefore the estimated values of $G_{c}$ and $G_{N}^{0}$ are inevitably distorted by numerical error of MC algorithm. This we need to take into account when analyzing the results of sensitivity analysis.

Since conversion and polymer molar mass distribution (MMD) evolve in time during polymerization in batch reactor, one can define several ways of measuring the model outputs (e.g., values at given time, average of values over a period of time). Here we are concerned with two different scenarios. In Scenario I we will observe values of the output states at reaction time $t_{I}=10 \mathrm{~min}$, when the conversion of monomer reaches value of $\sim 90 \%$ (based on nominal values of model inputs, cf. Table 3). In Scenario II, we will measure output values at $t_{I I}=2$ hours, i.e., sufficiently long time to reach the equilibrium conversion. Thus, in Scenario I we focus more on the dynamics of the reaction, while Scenario II reflects the resulting 
product properties rather than the polymerization dynamics. For the sake of brevity, analysis of Scenario II (which is of lower importance for industrial practice) is presented only in Supplementary Information.

Sobol's method needs much higher number of model evaluations than Morris screening, therefore only the method of Morris is used for sensitivity analysis of the whole computational framework (including prediction of rheological properties), while Sobol's algorithm is employed to assess only outputs of the deterministic simulation $\left(X, M_{n}, D P I\right)$ which are calculated very fast. Number of particles $N_{p}$ involved in micro-scale (MC) model of polymerization was set to $10^{4}$ in all simulations.

Table 3. List of input model parameters values (and their range) used in local and global sensitivity analysis.

\begin{tabular}{|c|c|c|c|c|c|c|}
\hline Description & Symbol & Unit & $\begin{array}{l}\text { Nominal } \\
\text { value }\end{array}$ & Uncertainty & $\begin{array}{l}\text { Lower } \\
\text { bound }\end{array}$ & $\begin{array}{l}\text { Upper } \\
\text { bound }\end{array}$ \\
\hline Reaction temperature & $T$ & ${ }^{\circ} \mathrm{C}$ & 160.0 & $\pm 0.5 \%$ & 159.2 & 160.8 \\
\hline $\begin{array}{l}\text { Initial monomer to catalyst } \\
\text { concentration ratio }\end{array}$ & {$\left[M_{0}\right] /\left[C_{0}\right]$} & - & $1.0 \times 10^{4}$ & $\pm 5.0 \%$ & $9.5 \times 10^{3}$ & $10.5 \times 10^{3}$ \\
\hline $\begin{array}{l}\text { Initial co-catalyst to } \\
\text { catalyst concentration ratio }\end{array}$ & & & 60 & $\pm 5.0 \%$ & 57 & 63 \\
\hline $\begin{array}{l}\text { Initial acid to catalyst } \\
\text { concentration ratio }\end{array}$ & & & 0.360 & $\pm 5.0 \%$ & 0.342 & 0.378 \\
\hline $\begin{array}{l}\text { Activation rate coefficient } \\
\text { Pre-exponential factor } \\
\text { of propagation }\end{array}$ & $k_{a 1}$ & $\mathrm{~L} / \mathrm{mol} / \mathrm{h}$ & $\begin{array}{l}1.0 \times 10^{6} \\
7.40 \times 10^{11}\end{array}$ & $\pm 3.6 \%$ & $7.13 \times 10^{11}$ & $7.67 \times 10^{11}$ \\
\hline $\begin{array}{l}\text { Activation energy } \\
\text { of propagation }\end{array}$ & $E_{a, p}$ & $\mathrm{~kJ} / \mathrm{mol}$ & 63.30 & $\pm 3.6 \%$ & 61.02 & 65.58 \\
\hline Heat of polymerization & $\Delta H_{l c}$ & $\mathrm{~kJ} / \mathrm{mol}$ & -23.3 & $\pm 6.4 \%$ & -24.79 & -21.81 \\
\hline Entropy of polymerization & $\Delta S_{l c}$ & $\mathrm{~J} / \mathrm{mol} / \mathrm{K}$ & -22.0 & $\pm 14.5 \%$ & -25.19 & -18.81 \\
\hline $\begin{array}{l}\text { Chain transfer rate } \\
\text { coefficient }\end{array}$ & $k_{s}$ & $\mathrm{~L} / \mathrm{mol} / \mathrm{h}$ & $1.0 \times 10^{6}$ & $\pm 5.0 \%$ & $9.5 \times 10^{5}$ & $10.5 \times 10^{5}$ \\
\hline
\end{tabular}


Pre-exponential factor of transesterification
$k_{t e 0}$
$\mathrm{L} / \mathrm{mol} / \mathrm{h} \quad 3.38 \times 10^{11} \quad \pm 6.9 \%$
$3.15 \times 10^{11} \quad 3.61 \times 10^{11}$

Activation energy

of transesterification

$\begin{array}{llllll}E_{a, t e} & \mathrm{~kJ} / \mathrm{mol} & 83.30 & \pm 6.9 \% & 77.55 & 89.05\end{array}$

Pre-exponential factor

of chain scission

$\begin{array}{llllll}k_{d e 0} & \mathrm{~h}^{-1} & 1.69 \times 10^{8} & \pm 17.0 \% & 1.40 \times 10^{8} & 1.98 \times 10^{8}\end{array}$

Activation energy

of scission

$E_{a, d e}$

$\mathrm{kJ} / \mathrm{mol}$

101.50

$\pm 17.0 \%$

84.25

118.76

\begin{tabular}{lcccccc}
\hline Polymer melt temperature & $T_{m}$ & ${ }^{\circ} \mathrm{C}$ & 200.0 & $\pm 0.5 \%$ & 199.0 & 201.0 \\
Polymer melt density & $\rho_{m}$ & $\mathrm{~kg} / \mathrm{m}^{3}$ & 1090.0 & $\pm 5.0 \%$ & 1035.5 & 1144.5 \\
Entanglement time & $\tau_{e}$ & $\mathrm{~s}$ & $9.0 \times 10^{-7}$ & $\pm 10.0 \%$ & $8.1 \times 10^{-7}$ & $9.9 \times 10^{-7}$ \\
Number of monomer units & & & & & & \\
between entanglements & $N_{e}$ & - & 65.0 & $\pm 10.0 \%$ & 58.5 & 71.5 \\
& & & & & &
\end{tabular}

When assessing significance of the model inputs based on results of the elementary effects (Morris') method, one should always take into account values of both mean and variance (or standard deviation) of the elementary effects distribution defined by Eqs. (19) and (21). This is typically done by plotting standard deviation versus mean together with two lines corresponding to $\mathrm{Mean}_{i}= \pm 2 \mathrm{SEM}_{i}$ for all model parameters $i=1 \ldots k$ [79], with $\mathrm{SEM}_{i}=S_{i} / r^{1 / 2}$ being the standard error of the mean (cf. Figure 9). Therefore, if the coordinates for input $i$ lie outside of the wedge formed by these two lines, it is a significant evidence of $i$ being an influential parameter. Moreover, distance of the input's coordinates from the zero point $(0 ; 0)$ is proportional to its importance with respect to model output. In contrast to Sobol sensitivity indices, means of elementary effects $\mu_{, i}$ (horizontal axis in Figure 9) indicate not only magnitude, but also direction (positive or negative) of the input's impact on the model output.

During the final stage of the polymerization when the chain growth still takes place, by far the most influential parameter for conversion is the activation energy of propagation. Generally, activation 
energies are more influential than pre-exponential factors due to their appearance in the exponential term of Arrhenius equation. Also the sign of the elementary effects mean is correct: increasing $E_{a, p}$ results in slower propagation and thus decrease in conversion. The effect of temperature on conversion is relatively small, but we have to bear in mind that temperature has also the lowest uncertainty from all model inputs. $E_{a, p}$ affects significantly also average chain length (reflected in $M_{n}$ ). $M_{n}$ is also influenced by initial concentration of cocatalyst which actually defines the number of reacting (active and dormant) chains in the reaction mixture, thereby directly influencing their average length. Probably the most influential parameter for $M_{n}$ is the activation energy of random chain scission $E_{a, d e}$. The phenomenon of chain scission (i.e., decrease in molecular weight) at high temperatures has been reported in the literature $[93,94,95]$ and becomes significant for longer chains, i.e., when conversion approaches its equilibrium value and afterwards. Contrary to the case of $E_{a, p}, E_{a, d e}$ is positioned in the right half (with positive values of mean) of $M_{n}$ plot in Fig. 9, because increase in activation barrier for chain scission actually allows chains to grow longer. Obviously, the polydispersity index $P D I$ is extremely sensitive to the intermolecular transesterification (activation energy $E_{a, t e}$. This reaction step (sometimes described as "re-shuffling") is responsible for broadening of the polymer MMD, i.e., increase of $P D I[96]$, therefore the negative sign of the mean in the plot. Chain scission $\left(E_{a, d e}\right)$ acts in a similar way but with smaller effect.

Input coordinates in the plots for crossover and plateau moduli $G_{c}$ and $G_{N}^{0}$, resp., seem to be more "noisy" at first sight. There are no zero values and most of the inputs are placed inside the wedge representing statistical error: this is probably caused by an "artificial sensitivity" introduced by statistical error of Monte Carlo simulation. Generally speaking (and after careful examination of the numerical values of means and standard deviations provided in Supplementary Information), $G_{c}$ and $G_{N}^{0}$ follow similar trends concerning the sensitivity to the reaction recipe and kinetic parameters, with the most influential inputs being those related to propagation $\left(E_{a, p}\right)$, transesterification $\left(E_{a, t e}\right)$ and scission reaction steps $\left(E_{a, d e}\right)$. Narrowing MMD should result in an increase of $G_{c}[69 ; 97]$ : for negative effect on $P D I$ a positive effect of $G_{c}$ should be expected, therefore the difference in signs observed for parameters $E_{a, t e}$ and $E_{a, d e}$ is a bit surprising. However, the two elementary reactions have different effect on the polymer MMD: transesterification dramatically 
affects polydispersity (via $M_{w}$ ), while retaining $M_{n}$ almost intact; chain scission, on the other hand, influences $P D I$ much less when compared to $M_{n}$. Among the inputs to BoB software, only the entanglement time $\tau_{e}$ is found outside the wedge in the plot for plateau modulus. Unfortunately, this input is typically highly uncertain, as it cannot be determined directly by any experimental method.

Last but not least, model sensitivity analysis can facilitate process of linking reaction conditions to the product application properties: for instance, PLLA molding and extrusion can be made easier by lowering crossover modulus $G_{c}$, which can be achieved either by decreasing reaction temperature and concentration of co-catalyst, or by increasing monomer to catalyst ratio, or by combination of all these actions.
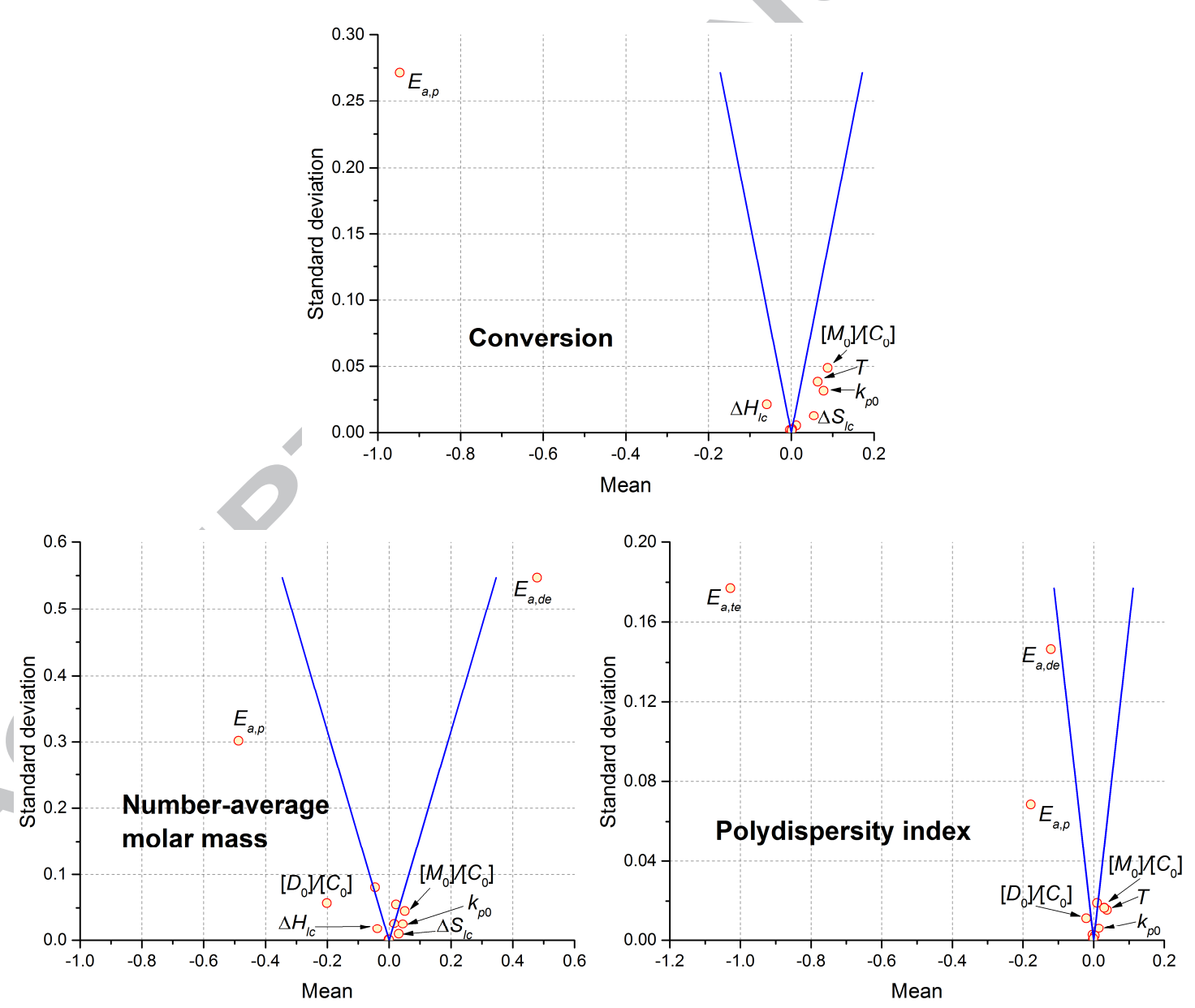

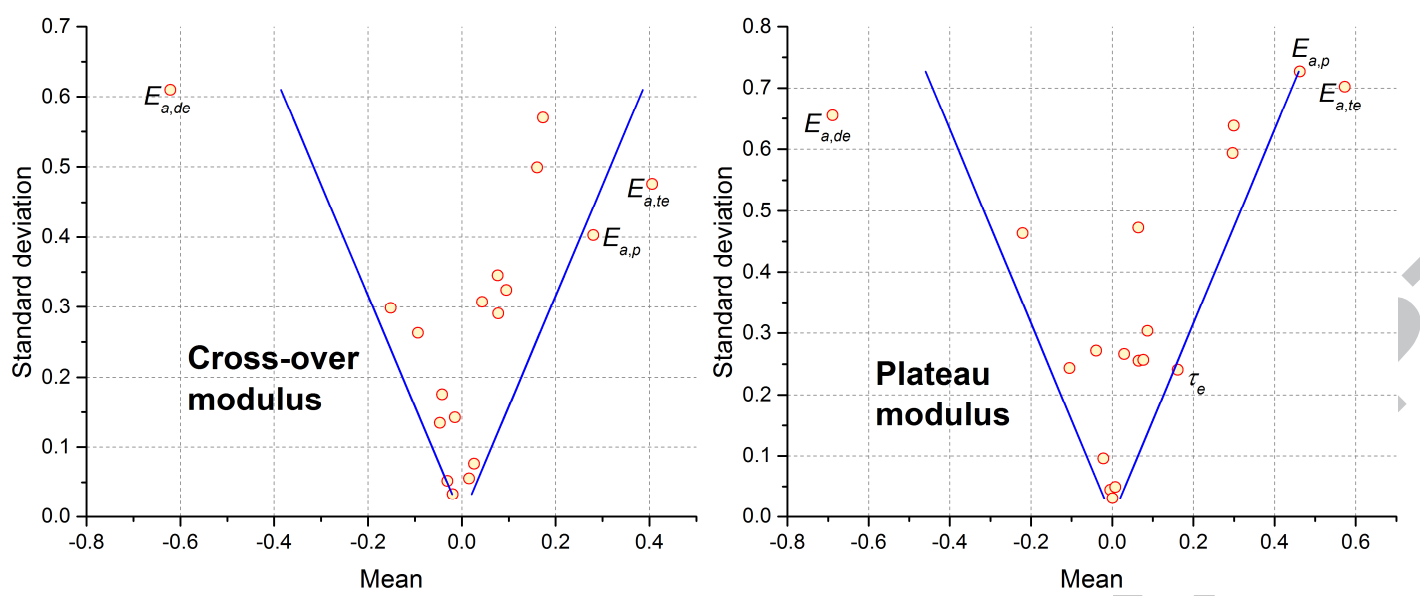

Figure 9. Estimated mean and standard deviation of the distribution of elementary effects of the model inputs on five different model outputs. The two blue lines drawn in each subplot correspond to $\mathrm{Mean}_{i}=$ $\pm 2 \mathrm{SEM}_{i}$ (see text). Original values of the means and standard deviations of the elementary effects distribution are presented in Supplementary Information.

First order sensitivity indices $S_{i}$ predicted by Sobol's method (available in Supplementary Information) provide information only about parameter significance, since they have all positive values. From their relative ranking presented in Figure 10, it is evident that their magnitude corresponds very nicely to the absolute values of the means of elementary effects distribution, which were estimated via significantly lower number of model evaluations. On the other hand, by means of total sensitivity indices $S_{T_{i}}$, method of Sobol provides information not only about the first order effect of the model inputs, but also about the total effect which involves contribution of the inputs' mutual interactions. In our study, the values of $S_{T_{i}}$ follow closely those of $S_{i}$ and the highest sum of $S_{T_{i}}$ over the input parameters (obtained for the case of $P D I$ in Scenario II) reached the value of 1.093 . This indicates that the variation in model outputs is driven primarily by the first order effects of the inputs with the interaction terms responsible in the worst case for only $9.3 \%$ of the model output variance. 


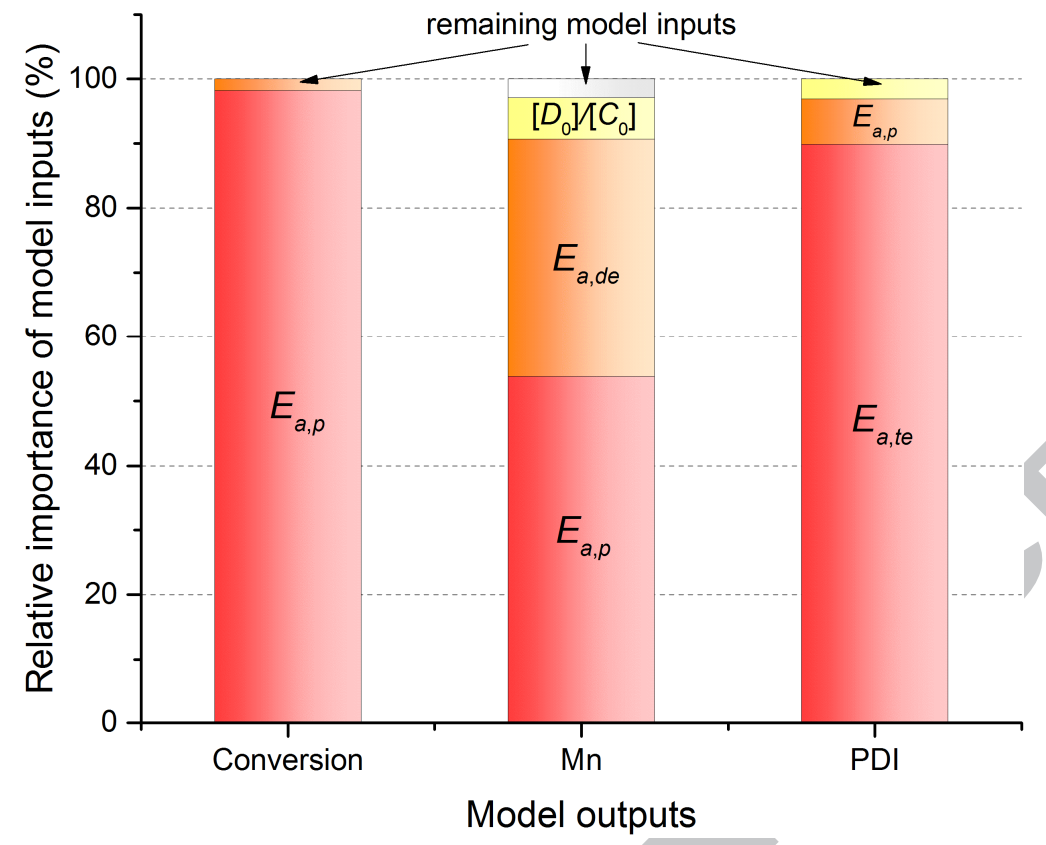

Figure 10. Relative importance of model input parameters on conversion, number-average molar mass $\left(M_{n}\right)$ and polydispersity index (PDI) calculated from first-order Sobol sensitivity indices $S_{i}$.

\section{Conclusions and Future Work}

This paper deals with the development of the modeling framework allowing mapping between reaction conditions during the L,L-lactide ring opening polymerization (ROP) and the application properties of the product represented by detailed molecular architecture and rheological characteristics. The conclusions of our work can be summarized in the following points:

- The deterministic model of L,L-lactide ROP experimentally validated at wide range of operating conditions was taken from the literature and extended by hybrid Monte Carlo algorithm and publicly available software for rheological estimations in order to expand the palette of predicted properties to full molar mass distribution (MMD) and viscosity of poly-(L-lactide) (PLLA) melt.

- The developed predictive framework was validated by experimental rheological characteristics of three different industrial PLLA samples taken from the literature. The entanglement molecular 
weight of the samples was estimated as $4200-4700 \mathrm{~g} / \mathrm{mol}$ in agreement with values previously reported in literature.

- Computational efficiency of the developed framework was critically assessed and shows a potential for the use as a soft sensor for MMD and polymer melt viscosity in the industrial production of PLLA.

- Model sensitivity analysis indicated that the reaction temperature and co-catalyst to catalyst ratio have positive impact on the crossover and plateau moduli of PLLA melt. Sobol index showed that up to $60 \%$ of variability in polymer polydispersity index at the final stage of polymerization can be explained by the intermolecular transesterification reaction.

In the future we plan to explore surrogate modeling techniques (e.g., Kriging interpolation, polynomial chaos expansion) to further enhance the computational efficiency of the developed model. In addition to that, the polymerization kinetic scheme will be extended by the presence of multifunctional co-catalysts resulting in a product with branched (nonlinear) architecture. This would allow for direct in silico tailoring of PLLA-based materials application properties via modification of reaction conditions and branch-on-branch architecture of the product.

Acknowledgement. The authors are grateful to Prof. Giuseppe Storti (ETH Zurich) for discussion of parametric uncertainties presented in the paper he co-authored [21].

\section{References}

[1] A consortium led by E4tech (UK) Ltd, From the Sugar Platform to biofuels and biochemicals - Final report for the European Commission Directorate-General Energy, Report No. ENER/C2/423-2012/SI2.673791, 2015.

[2] R. Auras, L.-T. Lim, S.E.M. Selke, H. Tsuji, Poly(lactic acid): Synthesis, Structures, Properties, Processing, and Applications, Wiley, New Jersey, USA, 2010.

[3] A.R. Kakroodi, Y. Kazemi, M. Nofar, C.B. Park, Tailoring poly(lactic acid) for packaging applications via the production of fully bio-based in situ microfibrillar composite films, Chem. Eng. J., 308 (2017)772-782. 
[4] S. Farah, D.G. Anderson, R. Langer, Physical and mechanical properties of PLLA, and their functions in widespread applications - A comprehensive review, Adv. Drug Deliver. Rev. 107 (2016) 367-392.

[5] A. Ameli, M. Nofar, D. Jahani, G. Rizvi, C.B. Park, Development of high void fraction polylactide composite foams using injection molding: Crystallization and foaming behaviors, Chem. Eng. J. 262 (2015) 78-87.

[6] P. Zhang, R. Tian, R. Lv, B. Na, Q. Liu, Water-permeable polylactide blend membranes for hydrophilicity-based separation, Chem. Eng. J. 269 (2015) 180-185.

[7] L.T. Sin, A.R. Rahmat, W.A.W.A. Rahman, Polylactic Acid: PLA Biopolymer Technology and Applications, William Andrew, 2012.

[8] M. Carus, W. Baltus, D. Carrez, H. Kaeb, J. Ravenstijn, S. Zepnik, Market Study on Bio-based Polymers in the WorldCapacities, Production and Applications: Satus Quo and Trends Towards 2020, nova-Institut GmbH, 2013.

[9] R. Datta, M. Henry, Lactic acid: recent advances in products, processes and technologies - a review, J. Chem. Technol. Biot. 81 (2006) 1119-1129.

[10] M. Jamshidian, E.A. Tehrany, M. Imran, M. Jacquot, S. Desobry, Poly-Lactic Acid: Production, Applications, Nanocomposites, and Release Studies, Compr. Rev. Food. Sci. F. 9 (2010) 552-571.

[11] A. Finne, A.-C. Albertsson, Controlled Synthesis of Star-Shaped L-Lactide Polymers Using New Spirocyclic Tin Initiators , Biomacromolecules 3 (2002) 684-690.

[12] K. Numata, R.K. Srivastava, A. Finne-Wistrand, A.-C. Albertsson, Y. Doi, H. Abe, Branched Poly(lactide) Synthesized by Enzymatic Polymerization: Effects of Molecular Branches and Stereochemistry on Enzymatic Degradation and Alkaline Hydrolysis, Biomacromolecules 8 (2007) 3115-3125.

[13] B.-K. Chen, T.-Y. Wu, Y.-M. Chang, A.F. Chen, Ductile polylactic acid prepared with ionic liquids, Chem. Eng. J. 215-216 (2013) 886-893.

[14] X. Zhang, D.A. MacDonald, M.F.A. Goosen, K.B. McAuley, Mechanism of lactide polymerization, in the presence of stannous octoate: The effect of hydroxyl and carboxylic acid substances, J. Polym. Sci. Al 32 (1994) 2965-2970.

[15] J.J. Kolstad, Crystallization Kinetics of Poly(L-lactide-co-meso-lactide), J. Appl. Polym. Sci. 62 (1996) 1079-1091.

[16] H.R. Kricherdolf, I. Kreiser-Saunders, A. Stricker, Polylactones 48. SnOct 2 -Initiated Polymerizations of Lactide: A Mechanistic Study, Macromolecules 33 (2000) 702-709.

[17] A. Kowalski, A. Duda, S. Penczek, Kinetics and Mechanism of Cyclic Esters Polymerization Initiated with Tin(II) Octoate. 3. Polymerization of L,L-Dilactide, Macromolecules 33 (2000) 7359-7370. 
[18] Y. Yu, G. Storti, M. Morbidelli, Ring-Opening Polymerization of L,L-Lactide: Kinetic and Modeling Study, Macromolecules 42 (2009) 8187-8197.

[19] J.-P. Puaux, I. Banu, I. Nagy, G. Bozga, A Study of L-Lactide Ring-Opening Polymerization Kinetics, Macromol. Symp. 259 (2007) 318-326.

[20] R. Mehta, V. Kumar, S.N. Upadhyay, Mathematical Modeling of the Poly(lactic acid) Ring-Opening Polymerization using Stannous Octoate as a Catalyst, Polym.-Plast. Technol. 46 (2007) 933-937.

[21] Y. Yu, G. Storti, M. Morbidelli, Kinetics of Ring-Opening Polymerization of L,L-Lactide, Ind. Eng. Chem. Res. 50 (2011) $7927-$ 7940.

[22] Y. Yu, G. Storti, M. Morbidelli, Modeling of Molecular Weight Distribution in Ring-Opening Polymerization of L,L-Lactide, Ind. Eng. Chem. Res. 53 (2014) 7333-7342.

[23] D. Refinetti, A. Sonzogni, F. Manenti, N.M.N. Lima, L.Z. Linan, R.M. Filho, Modeling and Simulation of Poly(L-Lactide) Polymerization in Batch Reactor, Chem. Engineer. Trans. 37 (2014) 691-696.

[24] S. Sosnowski, P. Lewinski, L-Lactide polymerization catalyzed by tin(II) 2-ethyl-hexanoate. A deeper look at chain transfer reactions, Polym. Chem. 6 (2015) 6292-6296.

[25] F. Weng, X. Li, Y. Wang, W.-J. Wang, S.J. Severtson, Kinetics and Modeling of Ring-Opening Copolymerization of L-Lactide and $\varepsilon$-Caprolactone, Macromol. React. Eng. 9 (2015) 535-544.

[26] D.R. D’hooge, M.-F. Reyniers, G.B. Marin, The Crucial Role of Diffusional Limitations in Controlled Radical Polymerization, Macromol. React. Eng. 7 (2013) 362-379.

[27] P. Pladis, K. Karidi, T. Mantourlias, C. Kiparissides, An Experimental and Theoretical Investigation of the Ring-Opening Polymerization of L,L-Lactide, Macromol. React. Eng. 8 (2014) 813-825.

[28] K. Karidi, P. Pladis, C. Kiparissides, A Theoretical and Experimental Kinetic Investigation of the ROP of L,L-Lactide in the Presence of Polyalcohols, Macromol. Symp. 333 (2013) 206-215.

[29] D. Meimaroglou, P. Pladis, C. Kiparissides, Dynamic Monte Carlo Simulation of the L,L-Lactide Ring-Opening Polymerization, Macromol. React. Eng. 11 (2017) 1600039.

[30] J.R. Dorgan, Rheology of Poly(lactic acid), in: R. Auras, L.-T. Lim, S.E.M. Selke, H. Tsuji (Eds.), Poly(lactic acid): Synthesis, Structures, Properties, Processing, and Applications, Wiley, New Jersey, 2010, pp. 125-139.

[31] J.J. Cooper-White, M.E. Mackay, Rheological Properties of Poly(lactides). Effect of Molecular Weight and Temperature on the Viscoelasticity of Poly(l-lactic acid), J. Polym. Sci. Pol. Phys. 37 (1999) 1803-1814. 
[32] J.R. Dorgan, JS. Williams, D.N. Lewis, Melt rheology of poly(lactic acid): Entanglement and chain architecture effects, $J$.

Rheol. 43 (1999) 1141-1155.

[33] J.R. Dorgan, H. Lehermeier, M. Mang, Thermal and Rheological Properties of Commercial-Grade Poly(Lactic Acid)s, J. Polym. Environ. 8 (2000) 1-9.

[34] H.J. Lehermeier, J.R. Dorgan, Melt Rheology of Poly(lactic acid): Consequences of Blending Chain Architectures, Polym. Eng. Sci. 41 (2001) 2172-2184.

[35] J.R. Dorgan, J. Janzen, D.M. Knauss, S.B. Hait, B.R. Limoges, M.H. Hutchinson, Fundamental Solution and Single-Chain Properties of Polylactides, J. Polym. Sci. Pol. Phys. 43 (2005) 3100-3111.

[36] A. Saltelli, M. Ratto, T. Andres, F. Campolongo, J. Cariboni, D. Gatelli, M. Saisana, S. Tarantola, Global Sensitivity Analysis. The Primer, Wiley, Chichester, UK, 2008.

[37] I.M. Thomas, C. Kiparissides, Sensitivity Analysis of a Batch Polymerization Reactor, J. Appl.Polym. Sci. 29 (1984) $2195-2204$.

[38] M. Atiqullah, M.M. Hassan, S.A. Beg, Influence of the gel effect and backmixing on the dynamics and rate parameters of bulk copolymerization in a tubular reactor - theoretical analysis, Chem. Eng. J. 48 (1992) 71-81.

[39] A. Flores-Tlacuahuac, E. Saldivar-Guerra, R. Guerrero-Santos, Dynamic Modelling, Nonlinear Parameter Fitting and Sensitivity Analysis of a Living Free-Radical Polymerization Reactor, in: S.P. Asprey, S. Machietto (Eds.), Dynamic Model Development:

Methods, Theory and Applications, Elsevier Science B.V., 2003, pp. 21-39.

[40] J. Lange, N. Davidenko, R. Sastre, Kinetic Study of the Thermopolymerization of Furfuryl Methacrylate in Bulk by Mathematical Modeling. Part A: Simulation of Experimental Data and Sensitivity Analysis of Kinetic Parameters, Macromol. Theor. Simul. 18 (2009) 511-527.

[41] V. Liotta, E.D. Sudol, M.S. El-Aasser, C. Georgakis, On-Line Monitoring, Modeling and Model Validation of Semibatch Emulsion Polymerization in an Automated Reactor Control Facility, J. Polym. Sci. Al 36 (1998) 1553-1571.

[42] A.L. Polic, L.M.F. Lona, T.A. Duever, A. Penlidis, A Protocol for the Estimation of Parameters in Process Models: Case Studies with Polymerization Scenarios, Macromol. Theor. Simul. 13 (2004) 115-132.

[43] R. Li, M.A. Henson, M.J. Kurtz, Selection of Model Parameters for Off-Line Parameter Estimation, IEEE T. Contr. Syst. T. 12 (2004) 402-412.

[44] B. Benyahia, M.A. Latifi, C. Fronteix, F. Pla, Emulsion copolymerization of styrene and butyl acrylate in the presence of a chain transfer agent. Part 2: Parameters estimability and confidence regions, Chem. Eng. Sci. 90 (2013) 110-118. 
[45] A.D. Bojarski, C.R. Alvarez, L. Puigjanger, Dealing with Uncertainty in Polymer Manufacturing by Using Linear Regression Metrics and Sensitivity Analysis, in: $19^{\text {th }}$ European Symposium on Computer Aided Process Engineering, Volume 26, Elsevier Science, 2009.

[46] S. Massebeuf, C. Fonteix, S. Hoppe, F. Pla, Development of New Concepts for the Control of Polymerization Processes: Multiobjective Optimization and Decision Engineering. I. Application to Emulsion Homopolymerization of Styrene, J. Appl. Polym. Sci. 87 (2003) 2383-2396.

[47] S.K. Fierens, D.R. D’hooge, P.H.M. Van Steenberge, M.-F. Reyniers, G.B. Marin, Exploring the Full Potential of Reversible Deactivation Radical Polymerization Using Pareto-Optimal Fronts, Polymers 7 (2015) 655-679.

[48] K.-D. Hungenberg, W.F. Reed, Online Monitoring, Control and Optimization of Polymer Reactions and Processes, Macromol. React. Eng. 11 (2017) 1700030.

[49] B. Colegrove, K. Deshpande, R. Harner, L. Mikolajczyk, S.K. Stephenson, J.D. Tate, J. Weston, Use of Online Spectroscopy to Control Polymerization in Industrial Processes, Macromol. React. Eng. 11 (2017) 1600056.

[50] A. Zubov, O. Naeem, S.O. Hauger, A. Bouaswaig, F. Gjertsen, P. Singstad, K.-D. Hungenberg, J. Kosek, Bringing the On-Line Control and Optimization of Semibatch Emulsion Copolymerization to the Pilot Plant, Macromol. React. Eng. 11 (2017) 1700014.

[51] C. Das, N.J. Inkson, D.J. Read, M.A. Kelmanson, T.C.B. McLeish, Computational linear rheology of general branch-on-branch polymers, J. Rheol. 50 (2006) 207-235.

[52] Q.H. Zeng, A.B. Yu, G.Q. Lu, Multiscale modeling and simulation of polymer nanocomposites, Prog. Polym. Sci. 33 (2008) 191-269.

[53] D.R. D’hooge, P.H.M. Van Steenberge, M.-F. Reyniers, G.B. Marin, The strength of multi-scale modeling to unveil the complexity of radical polymerization, Prog. Polym. Sci. 58 (2016) 59-89.

[54] P. Ferkl, M. Karimi, D.L. Marchisio, J. Kosek, Multi-scale modeling of expanding polyurethane foams: Coupling macro- and bubble-scales, Chem. Eng. Sci. 148 (2016) 55-64.

[55] D.T. Gillespie, A General Method for Numerically Simulating the Stochastic Time Evolution of Coupled Chemical Reactions, J. Comput. Phys. 22 (1976) 403-434.

[56] A.C. Hindmarsh, ODEPACK, A Systematized Collection of ODE Solvers, in: R.S. Stepleman, M. Carver, R. Peskin, W.F. Ames, R. Vichnevetsky (Eds.), Scientific Computing: Applications of Mathematics and Computing to the Physical Sciences, NorthHolland, Amsterdam, 1983, pp. 55-64.

[57] D.R. Witzke, R. Narayan, J.J. Kolstad, Reversible Kinetics and Thermodynamics of the Homopolymerization of L-Lactide with 2-Ethylhexanoic Acid Tin(II) Salt, Macromolecules 30 (1997) 7075-7085. 
[58] R. Szymanski, On the determination of the ratios of the propagation rate constants on the basis of the MWD of copolymer chains: A new Monte Carlo algorithm, e-Polymers 9 (2009) 044.

[59] P.H.M. Van Steenberge, D.R. D’hooge, Y. Wang, M. Zhong, M.-F. Reyniers, D. Konkolewicz, , K. Matyjaszewski, G.B. Marin, Linear Gradient Quality of ATRP Copolymers, Macromolecules 45 (2012) 8519-8531.

[60] D. Meimaroglou, C. Kiparissides, Review of Monte Carlo Methods for the Prediction of Distributed Molecular and Morphological Polymer Properties, Ind. Eng. Chem. Res. 53 (2014) 8963-8979.

[61] D.R. D’hooge, P.H.M. Van Steenberge, P. Derboven, M.-F. Reyniers, G.B. Marin, Model-based design of the polymer microstructure: bridging the gap between polymer chemistry and engineering, Polym. Chem. 6 (2015) 7081-7096.

[62] A.L.T. Brandão, J.B.P. Soares, J.C. Pinto, A.L. Alberton, When Polymer Reaction Engineers Play Dice: Applications of Monte Carlo Models in PRE, Macromol. React. Eng. 9 (2015) 141-185.

[63] M.A. Parsa, I. Kozhan, M. Wulkow, R.A. Hutchinson, Modeling of Functional Group Distribution in Copolymerization: A Comparison of Deterministic and Stochastic Approaches, Macromol. Theory Simul. 23 (2014) 207-217.

[64] E. Neuhaus, T. Herrmann, I. Vittorias, D. Lilge, G. Mannebach, A. Gonioukh, M. Busch, Modeling the Polymeric Microstructure of LDPE in Tubular and Autoclave Reactors with a Coupled Deterministic and Stochastic Simulation Approach, Macromol. Theory Simul. 23 (2014) 415-428.

[65] A.K. Tripathi, D.C. Sundberg, A Hybrid Algorithm for Accurate and Efficient Monte Carlo Simulations of Free-Radical Polymerization Reactors, Macromol. Theory Simul. 24 (2015) 52-64.

[66] T.C.B. McLeish, Tube theory of entangled polymer dynamics, Adv. Phys. 51 (2002) 1379-1527.

[67] L.H. Sperling, Introduction to Physical Polymer Science, Wiley, New Jersey, USA, 2006.

[68] R.R. Rahalkar, Correlation between the crossover modulus and the molecular weight distribution using the Doi-Edwards theory of reputation and the Rouse theory, Rheol. Acta 28 (1989) 166-175.

[69] M. Fleissner, Characterization of Polymer Molecular Mass Distribution from Rheological Measurements, Makromol. Chem., Macomol. Symp. 61 (1992) 324-341.

[70] A.D. Gotsis, Branched Polyolefins, in: M. Kontopoulu (Ed.), Applied Polymer Rheology: Polymer Fluids with Industrial Applications, Wiley, New Jersey, 2012, pp.59-112.

[71] R.G. Larson, T. Sridhar, L.G. Leal, G.H. McKinley, A.E. Likhtman, T.C.B. McLeish, Definitions of entanglement spacing and time constants in the tube model, J. Rheol. 47 (2003) 809-818.

[72] P.-G. de Gennes, Reptation of a Polymer Chain in the Presence of Fixed Obstacles, J. Chem. Phys. 55 (1971) $572-579$. 
[73] M. Doi, S.F. Edwards, The Theory of Polymer Dynamics, Oxford University Press, Oxford, UK, 1986.

[74] D.J. Read, D. Auhl, C. Das, J. den Doelder, M. Kapnistos, I. Vittorias, T.C.B. McLeish, Linking Models of Polymerization and Dynamics to Predict Branched Polymer Structure and Flow, Science 333 (2011) 1871-1874.

[75] C. Das, D.J. Read, D. Auhl, M. Kapnistos, J. den Doelder, I. Vittorias, T.C.B. McLeish, Numerical prediction of nonlinear rheology of branched polymer melts, J. Rheol. 58 (2014) 737-577.

[76] C. Liu, J. He, E. van Ruymbeke, R. Keunings, C. Bailly, Evaluation of different methods for the determination of the plateau modulus and the entanglement molecular weight, Polymer 47 (2006) 4461-4479.

[77] S. Wu, Chain structure and entanglement, J. Polym. Sci. Pol. Phys. 27 (1989) 723-741.

[78] G. Sin, K.V. Gernaey, Data Handling and Parameter Estimation, in: M.C.M. van Loosdrecht, P.H. Nielsen, C.M. LopezVazquez, D. Brdjanovic (Eds.), Experimental Methods in Wastewater Treatment, IWA Publishing, London, UK, 2016.

[79] M.D. Morris, Factorial Sampling Plans for Preliminary Computational Experiments, Technometrics 33 (1991) 161-174.

[80] F. Campolongo, S. Tarantola, A. Saltelli, Tackling quantitatively large dimensionality problems, Comput. Phys. Commun. 117 (1999) $75-85$.

[81] A. Saltelli, K. Chan, M. Scott, Sensitivity Analysis, Wiley, New York, 2000.

[82] I.M. Sobol', Sensitivity Estimates for Nonlinear Mathematical Models, MMCE 1 (1993), 407-414.

[83] T. Homma, A. Saltelli, Importance measures in global sensitivity analysis of nonlinear models, Reliab. Eng. Syst. Safe. 52 (1996) 1-17.

[84] A. Saltelli, S. Tarantola, On the Relative Importance of Input Factors, in Mathematical Models: Safety Assessment for Nuclear Waste Disposal, J. Am. Stat. Assoc. 97 (2002) 702-709.

[85] A. Saltelli, P. Annoni, I. Azzini, F. Campolongo, M. Ratto, S. Tarantola, Variance based sensitivity analysis of model output. Design and estimator for the total sensitivity index, Comput. Phys. Commun. 181 (2010) 259-270.

[86] M.J.W. Jansen, Analysis of variance designs for model output, Comput. Phys. Commun. 117 (1999) 35-43.

[87] F. Campolongo, J. Cariboni, A. Saltelli, An effective screening design for sensitivity analysis of large models, Environ. Modell. Softw. 22 (2007) 1509-1518.

[88] P. Bratley, B.L. Fox, Algorithm 659 Implementing Sobol's Quasirandom Sequence Generator, ACM T. Math. Software 14 (1988) 88-100.

[89] J. Matousek, On the L2-Discrepancy for Anchored Boxes, J. of Complexity, 14 (1998) 527-556. 
[90] C. Pattamaprom, R.G. Larson, T.J. Van Dyke, Quantitative predictions of linear viscoelastic rheological properties of entangled polymers, Rheol. Acta 39 (2000) 517-531.

[91] M. Drache, G. Drache, Simulating Controlled Radical Polymerizations with mcPolymer - A Monte Carlo Approach, PolymersBasel 4 (2012) 1416-1442.

[92] R.H. Colby, M. Rubinstein, Two-Parameter Scaling for Polymers in $\theta$ Solvents, Macromolecules 23 (1990) 2753-2757.

[93] Y. Wang, B. Steinhoff, C. Brinkmann, I. Alig, In-line monitoring of the thermal degradation of poly(L-lactic acid) during melt extrusion by UV-vis spectroscopy, Polymer 49 (2008) 1257-1265.

[94] H. Nishida, T. Mori, S. Hoshihara, Y. Fan, Y. Shirai, T. Endo, Effect of tin on poly(L-lactic acid) pyrolysis, Polym. Degrad. Stabil. 81 (2003) 515-523.

[95] Y. Fan, H. Nishida, Y. Shirai, T. Endo, Thermal stability of poly(L-lactide): influence of end protection by acetyl group, Polym. Degrad. Stabil. 84 (2004) 143-149.

[96] R. Szymanski, Molecular weight distribution in living polymerization proceeding with reshuffling of polymer segments due to chain transfer to polymer with chain scission, 1 . Determination of $k_{p} / k_{t r}$ ratio from $\mathrm{DP}_{\mathrm{w}} / \mathrm{DP}_{\mathrm{n}}$ data. Ideal reproduction of polymer chain activities, Macromol. Theor. Simul. 7 (1998) 27-39.

[97] TA Instruments (A.J. Franck), Understanding Rheology of Thermoplastic Polymers.

http://www.tainstruments.com/pdf/literature/AAN013_V_1_U_Thermoplast.pdf, 2004 (accessed 26 ${ }^{\text {th }}$ September 2017). 
Highlights for manuscript "Multiscale Modeling of Poly(lactic acid) Production: From Reaction Conditions to Rheology of Polymer Melt":

- Multi-scale model of PLLA (poly[L-lactic acid]) production was developed.

- The developed framework allows fast prediction of polymer melt viscosity.

- The model was validated by rheological data measured for various PLLA samples.

- Comprehensive global sensitivity analysis of the model was performed. 